Xiuxiong Chen $\cdot$ Bing Wang

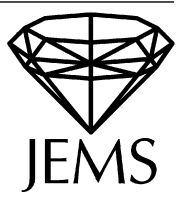

\title{
The Kähler Ricci flow on Fano manifolds (I)
}

Received March 9, 2011 and in revised form May 20, 2011

Abstract. We study the evolution of pluri-anticanonical line bundles $K_{M}^{-v}$ along the Kähler Ricci flow on a Fano manifold $M$. Under some special conditions, we show that the convergence of this flow is determined by the properties of the pluri-anticanonical divisors of $M$. For example, the Kähler Ricci flow on $M$ converges when $M$ is a Fano surface satisfying $c_{1}^{2}(M)=1$ or $c_{1}^{2}(M)=3$. Combined with the work of [CW1] and [CW2], this gives a Ricci flow proof of the Calabi conjecture on Fano surfaces with reductive automorphism groups. The original proof of this conjecture is due to Gang Tian [Tian90].

\section{Contents}

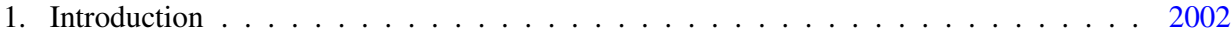

2. Estimates along the Kähler Ricci flow . . . . . . . . . . . . . . . . . . . . . 2007

2.1. Fundamentals of Kähler geometry . . . . . . . . . . . . . . . . . . . . . 2007

2.2. Known estimates along general Kähler Ricci flow . . . . . . . . . . . . . . . . 2008

2.3. Estimates along the tamed Kähler Ricci flow . . . . . . . . . . . . . . . . . . 2009

3. Pluri-anticanonical line bundles $K_{M}^{-v}$ and the tameness condition . . . . . . . . . 2016

3.1. Uniform bounds of holomorphic sections of $K_{M}^{-v} \ldots \ldots \ldots \ldots$. . . . . . . 2016

3.2. Convergence of holomorphic sections of $K_{M}^{-v} \ldots \ldots \ldots \ldots \ldots$. . . . . . 2023

3.3. Criteria for tameness . . . . . . . . . . . . . . . . . . 2027

4. The Kähler Ricci flow on Fano surfaces ． . . . . . . . . . . . . . . . . . . . . . . . . 2029

4.1. Convergence of 2-dimensional Kähler Ricci flow . . . . . . . . . . . . . . . 2029

4.2. Calculation of local $\alpha$-invariants . . . . . . . . . . . . . . . . . . . . . 2030

4.2.1. Local $\alpha$-invariants of holomorphic sections of $K_{M}^{-1} \ldots \ldots \ldots$. . . . . . 2030

4.2.2. Local $\alpha$-invariants of holomorphic sections of $K_{M}^{-v} \ldots \ldots \ldots$. . . . . 2032

References . . . . . . . . . . . . . . . . . . . . . . 2036

X. X. Chen: Department of Mathematics, University of Wisconsin-Madison,

Madison, WI 53706, USA; e-mail: xiu@math.wisc.edu

B. Wang: Department of Mathematics, Princeton University, Princeton, NJ 08544, USA;

e-mail: bingw@math.princeton.edu;

current address: Department of Mathematics, University of Wisconsin-Madison,

480 Lincoln Drive, Madison, WI 53706, USA; e-mail: bwang@math.wisc.edu 


\section{Introduction}

In this paper, we introduce new criteria for the convergence of the Kähler Ricci flow on general Fano manifolds. Moreover, we verify these criteria for the Kähler Ricci flow on the Fano surfaces $\mathbb{C P}^{2} \# 8 \overline{\mathbb{C P}}^{2}$ and $\mathbb{C P}^{2} \# 6 \overline{\mathbb{C P}}^{2}$. Consequently, we give a proof of the convergence of the Kähler Ricci flow on such Fano surfaces. The existence of KE (Kähler Einstein) metrics on these Fano surfaces follows as a corollary.

In Kähler geometry, a dominating problem is to prove the celebrated Calabi conjecture ([Ca]). It states that if $c_{1}(M)$ (the first Chern class of a Kähler manifold $M$ ) is positive, null or negative, then $M$ admits a KE metric. In 1976, the null case Calabi conjecture was proved by S. T. Yau. Around the same time, the negative case was proved independently by T. Aubin and S. T. Yau. However, the case of positive first Chern class is much more complicated. In [Ma], Matsushima showed that the reductivity of $\operatorname{Aut}(M)$ is a necessary condition for the existence of KE metric. In [Fu], A. Futaki introduced an algebraic invariant which vanishes if $M$ admits a KE metric. Around 1988, G. Tian [Tian90] proved the Calabi conjecture for Fano surfaces with reductive automorphism groups. Prior to Tian's work, there is a series of important works [Tian87], [TY], [Siu] where existence of KE metrics on some special complex surfaces were derived.

Let $(M, J)$ be a Fano manifold, $\left\{\omega_{t}, 0 \leq t<\infty\right\}$ be a one-parameter family of Kähler metric forms in $2 \pi c_{1}(M)$, and $g(t)=g_{t}$ be the metrics compatible with $\omega_{t}$ and $J$. Suppose $g(t)$ evolves along the Kähler Ricci flow. Let $K_{M}^{-1}$ be the anticanonical line bundle, and $h_{t}=\omega_{t}^{n}$ be the evolving metrics of $K_{M}^{-1}$. Let $v$ be a large integer such that $K_{M}^{-v}$ is very ample. In this paper, we study the evolution of the line bundles $\left(K_{M}^{-v}, h_{t}^{v}\right)$. Let $N_{v}=\operatorname{dim} H^{0}\left(K_{M}^{-v}\right)-1$, and $\left\{S_{v, \beta}^{t}\right\}_{\beta=0}^{N_{v}}$ be orthonormal holomorphic sections in $H^{0}\left(K_{M}^{-v}\right)$ with respect to the metrics $\omega_{t}$ and $h_{t}^{v}$, i.e.,

$$
\int_{M}\left\langle S_{v, \alpha}^{t}, S_{v, \beta}^{t}\right\rangle_{h_{t}^{v}} \omega_{t}^{n}=\delta_{\alpha \beta}, \quad 0 \leq \alpha, \beta \leq N_{v}
$$

It is easy to observe that

$$
F_{v}(x, t)=\frac{1}{v} \log \sum_{\beta=0}^{N_{v}}\left|S_{\nu, \beta}^{t}\right|_{h_{t}^{v}}^{2}(x)
$$

is a well defined function on $M \times[0, \infty)$ (independent of the choice of orthonormal basis). The Kähler Ricci flow $\left\{\left(M, \omega_{t}\right), 0 \leq t<\infty\right\}$ is said to be tamed by $v$ if $K_{M}^{-v}$ is very ample and $F_{v}(x, t)$ is a uniformly bounded function on $M \times[0, \infty)$. The flow is a tamed Kähler Ricci flow if it is tamed by some integer $v$.

For a tamed flow, we show that one can give criteria for the convergence of the flow by using some invariants defined in complex algebraic geometry.

Definition 1. Suppose $L$ is a line bundle over $M$ with Hermitian metric $h, S$ is a holomorphic section of $L, x \in M$. Define

$$
\alpha_{x}(S)=\sup \left\{\alpha \mid\|S\|_{h}^{-2 \alpha} \text { is locally integrable around } x\right\} .
$$

It is called the local $\alpha$-invariant of the section $S$ at point $x$. 
See [Tian90] and [Tian91] for more details about this definition. Note that $\alpha_{x}(S)$ is also called the singularity exponent ([DK]), log canonical threshold ([Chl]), etc. It is determined only by the singularity type of $Z(S)$. Therefore, if $S \in H^{0}\left(K_{M}^{-v}\right)$, then $\alpha_{x}(S)$ can only achieve finitely many possible values.

Definition 2. Let $G$ be a compact subgroup of $\operatorname{Aut}(M), \omega_{0} \in 2 \pi c_{1}(M)$ be a $G$-invariant metric, and $\mathscr{P}_{G, v, k}\left(M, \omega_{0}\right)$ be the collection of all $G$-invariant functions of the form $v^{-1} \log \left(\sum_{\beta=0}^{k-1}\left\|\tilde{S}_{v, \beta}\right\|_{h_{0}^{v}}^{2}\right)$, where $\left\{\tilde{S}_{\nu, \beta}\right\}_{\beta=0}^{k-1} \subset H^{0}\left(K_{M}^{-v}\right)\left(1 \leq k \leq N_{v}+1\right)$ satisfies

$$
\int_{M}\left\langle\tilde{S}_{\nu, \alpha}, \tilde{S}_{\nu, \beta}\right\rangle_{h^{\nu}} \omega_{0}^{n}=\delta_{\alpha \beta}, \quad 0 \leq \alpha, \beta \leq k-1 \leq N_{\nu} ; \quad h_{0}=\omega_{0}^{n} .
$$

The number $\sup \left\{\alpha \mid \sup _{\varphi \in \mathscr{P}_{G, v, k}} \int_{M} e^{-\alpha \varphi} \omega_{0}^{n}<\infty\right\}$ depends only on the class $\left[\omega_{0}\right]=$ $2 \pi c_{1}(M)$. We denote this number by $\alpha_{G, v, k}(M)$. If $G=\{\mathrm{id}\}$, we denote $\alpha_{\{\mathrm{id}\}, v, k}(M)$ by $\alpha_{v, k}(M)$.

It turns out that the values of $\alpha_{v, 1}$ and $\alpha_{\nu, 2}$ play important roles in the convergence of the tamed Kähler Ricci flow. Denote $\omega_{t}=\omega_{0}+\sqrt{-1} \partial \bar{\partial} \varphi(t)$ where $\varphi(t)$ is the Kähler potential. We have the following theorems.

Theorem 1. Suppose $\left\{\left(M^{n}, g(t)\right), 0 \leq t<\infty\right\}$ is a Kähler Ricci flow tamed by $v$. If $\alpha_{v, 1}>n /(n+1)$, then $\varphi$ is uniformly bounded along this flow. In particular, this flow converges to a KE metric exponentially fast.

Theorem 2. Suppose $\left\{\left(M^{n}, g(t)\right), 0 \leq t<\infty\right\}$ is a Kähler Ricci flow tamed by $v$. If

$$
\alpha_{v, 2}>\frac{n}{n+1} \quad \text { and } \quad \alpha_{v, 1}>\frac{1}{2-\frac{n-1}{(n+1) \alpha_{v, 2}}},
$$

then $\varphi$ is uniformly bounded along this flow. In particular, this flow converges to a KE metric exponentially fast.

In fact, if a Kähler Ricci flow is tamed by some large integer v, an easy argument (cf. Section 2.3) shows that the following strong partial $C^{0}$-estimate holds:

$$
\left.\left|\varphi(t)-\sup _{M} \varphi(t)-\frac{1}{v} \log \sum_{\beta=0}^{N_{v}}\right| \lambda_{\beta}(t) \tilde{S}_{\nu, \beta}^{t}\right|_{h_{0}^{v}} ^{2} \mid<C .
$$

Here $\left\{\lambda_{k}(t)\right\}_{k=0}^{N_{v}}$ are $N_{v}+1$ positive functions of time which satisfy

$$
0<\lambda_{0}(t) \leq \lambda_{1}(t) \leq \cdots \leq \lambda_{N_{v}}(t)=1
$$

$\left\{\tilde{S}_{v, \beta}^{t}\right\}_{\beta=0}^{N_{v}}$ is an orthonormal basis of $H^{0}\left(K_{M}^{-v}\right)$ under the fixed metric $\omega_{0}$. Intuitively, inequality (1) means that we can control $\operatorname{Osc}_{M} \varphi(t)$ by $v^{-1} \log \sum_{\beta=0}^{N_{v}}\left|\lambda_{\beta}(t) \tilde{S}_{\nu, \beta}^{t}\right|_{h_{0}^{v}}^{2}$ which only blows up along intersections of pluri-anticanonical divisors. Therefore, the estimate of $\varphi(t)$ is more or less determined by the properties of the pluri-anticanonical divisors.

In view of these theorems, it is important to check the following two conditions:

- The Kähler Ricci flow is tamed by a large integer $v$;

- $\alpha_{v, k}(k=1,2)$ are large enough. 
$\alpha_{\nu, k}$ can be calculated by algebraic geometry methods. The first condition is weak. We believe that it holds for every Kähler Ricci flow on a Fano manifold although we cannot prove this right now. However, under some extra conditions, we can check the first condition by applying the following theorem.

Theorem 3. Suppose $\left\{\left(M^{n}, g(t)\right), 0 \leq t<\infty\right\}$ is a Kähler Ricci flow satisfying the following conditions:

- Volume ratio bounded from above, i.e., there exists a constant $K$ such that

$$
\operatorname{Vol}_{g(t)}\left(B_{g(t)}(x, r)\right) \leq K r^{2 n}
$$

for every geodesic ball $B_{g(t)}(x, r)$ satisfying $r \leq 1$.

- Weak compactness, i.e., for every sequence $t_{i} \rightarrow \infty$, by passing to a subsequence if necessary, we have

$$
\left(M, g\left(t_{i}\right)\right) \stackrel{C^{\infty}}{\longrightarrow}(\hat{M}, \hat{g}),
$$

where $(\hat{M}, \hat{g})$ is a $Q$-Fano metric-normal variety, “ $\stackrel{C^{\infty}}{\longrightarrow}$ " means Cheeger-Gromov convergence, i.e., $\left(M, g\left(t_{i}\right)\right)$ converges to $(\hat{M}, \hat{g})$ in the Gromov-Hausdorff topology, and the convergence is in the smooth topology away from the singularities.

Then this flow is tamed.

In the case of Fano surfaces, Tian proved a similar theorem in order to solve the Kähler Einstein equation under a continuous path of complex structures. There are more estimates available in his approach since every metric there is a KE metric. In particular, Ricci curvature is constant. In our approach, more technical difficulties appear since Ricci curvature may be unbounded. The concept of Q-Fano variety was first defined in [DT]; it is a natural generalization of Fano manifold. A Q-Fano variety is an algebraic variety with a very ample line bundle whose restriction to the smooth part is the pluri-anticanonical line bundle. Let $\mathcal{P}$ be the singular part of the Q-Fano variety $\hat{M}$. We say $(\hat{M}, \hat{g})$ is metricnormal if $\hat{M}$ is a normal variety and the Minkowski codimension of $\mathcal{P}$ is not less than 4 with respect to the metric $\hat{g}$. In the proof of this theorem, Hörmander's $L^{2}$-estimate of the $\bar{\partial}$-operator, Perelman's fundamental estimates and the uniform control of Sobolev constants (cf. [Ye], [Zhq]) play crucial roles. Actually, Sobolev constants control and Perelman's estimates guarantee the uniform bound of $\left\||S|_{h_{t}^{v}}\right\|_{C^{0}(M)}$ and $\left\||\nabla S|_{h_{t}^{v}}\right\|_{C^{0}(M)}$ whenever $S$ is a unit norm holomorphic section in $H^{0}\left(K_{M}^{-v}\right)$. Hörmander's $L^{2}$-estimate of the $\bar{\partial}$-operator implies that the plurigenera are sequentially continuous. Therefore, for every fixed $\nu$, we have

$$
\lim _{i \rightarrow \infty} \inf _{x \in M} e^{\nu F_{\nu}\left(x, t_{i}\right)}=\lim _{i \rightarrow \infty} \inf _{x \in M} \sum_{\beta=0}^{N_{v}}\left|S_{\nu, \beta}^{t_{i}}\right|_{h_{t_{i}}^{v}}^{2}(x)=\inf _{x \in \hat{M}} \sum_{\beta=0}^{N_{v}}\left|\hat{S}_{\nu, \beta}\right|_{\hat{h}^{v}}^{2}(x) .
$$

This equation relates the tameness condition to the property of every limit space. If every limit space is a Q-Fano metric-normal variety, we know

$$
\inf _{x \in \hat{M}} \sum_{\beta=0}^{N_{v}}\left|\hat{S}_{v, \beta}\right|_{h_{t_{i}}^{v}}^{2}(x)>0
$$


for some $v$ depending on $\hat{M}$. Then a contradiction argument shows that $e^{v F_{v}}$ must be uniformly bounded from below for some large $v$. In other words, $F_{v}$ is uniformly bounded (the upper bound of $F_{v}$ is implied by the upper bound of $\left\||S|_{h_{t}^{v}}\right\|_{C^{0}(M)}$ ) and the flow is tamed.

As applications of Theorems 1-3, we can show the convergence of the Kähler Ricci flow on a Fano surface $M$ when $c_{1}^{2}(M) \leq 4$.

In [CW3], we proved the weak compactness of every 2-dimensional Kähler Ricci flow.

Lemma 1 ([CW3]). Suppose $\{(M, g(t)), 0 \leq t<\infty\}$ is a Kähler Ricci flow solution on a Fano surface. Then for every sequence $t_{i} \rightarrow \infty$, we have the Cheeger-Gromov convergence

$$
\left(M, g\left(t_{i}\right)\right) \stackrel{C^{\infty}}{\longrightarrow}(\hat{M}, \hat{g})
$$

where $(\hat{M}, \hat{g})$ is a KRS orbifold with finite singularities. In particular, $\hat{M}$ is a $Q$-Fano metric-normal variety.

Moreover, the volume ratio upper bound is proved in the process of proving weak compactness. Therefore, Theorem 3 and Lemma 1 imply that every 2-dimensional Kähler Ricci flow is tamed. We remark that, in an unpublished work (cf. [Se1]), Tian has pointed out earlier the sequential convergence of the 2-dimensional Kähler Ricci flow to Kähler Ricci soliton orbifolds under the Gromov-Hausdorff topology. Under the extra condition that Ricci curvature is uniformly bounded along the flow, Lemma 1 was proved by Natasa Sesum [Se1]. However, for our purpose of using Theorem 3, these convergence theorems are not sufficient (we need Cheeger-Gromov convergence without the Ricci curvature bound). In the course of proving this lemma, the fundamental works of G. Perelman (cf. [Pe]) on the Ricci flow (no-local-collapsing theorem, pseudo-locality theorem and contradiction arguments for canonical neighborhood theorem) play crucial roles. Under some natural geometric constraints, we prove an inverse pseudo-locality theorem and obtain estimates of volume ratios of geodesic balls. We found that the arguments of volume ratios in [TV1] and [TV2] are very enlightening.

In order to show the convergence of a 2-dimensional Kähler Ricci flow, we now only need to see that $\alpha_{v, k}(k=1,2)$ are large enough to satisfy the requirements of Theorem 1 or Theorem 2. If $c_{1}^{2}(M) \leq 4$, one can show that either Theorem 1 or Theorem 2 applies. However, the convergence of the Kähler Ricci flow on Fano surfaces $M$ was proved in [CW2] when $c_{1}^{2}(M)=2$ or $c_{1}^{2}(M)=4$. The only remaining cases are $c_{1}^{2}(M)=1$ and $c_{1}^{2}(M)=3$. So we concentrate on these two cases and prove the following lemma.

Lemma 2. Suppose $M$ is a Fano surface $v$ is any positive integer.

- If $c_{1}^{2}(M)=1$, then $\alpha_{v, 1} \geq 5 / 6$.

- If $c_{1}^{2}(M)=3$, then $\alpha_{v, 1} \geq 2 / 3$ and $\alpha_{v, 2}>2 / 3$.

Actually, the value of $\alpha_{v, 1}$ was calculated by Ivan Cheltsov (cf. [Chl]) for every Fano surface. The value of $\alpha_{v, 2}$ was also calculated for every cubic surface $\left(c_{1}^{2}(M)=3\right)$ by 
Yalong Shi (cf. [Shi]). For the convenience of the readers, we give an elementary proof at the end of this paper.

Therefore, Theorem 1 or Theorem 2 applies to show the existence of KE metrics on $M$ whenever $c_{1}^{2}(M)=1$ or 3 respectively. Combining this result with the results we proved in [CW1] and [CW2], we can give an alternative proof of the celebrated theorem of Tian.

Theorem ([Tian90]). A Fano surface $M$ admits a KE metric if and only if $\operatorname{Aut}(M)$ is reductive.

This solved a famous problem of Calabi for Fano surfaces [Ca]. This work of Tian clearly involves a deep understanding of many aspects of Kähler geometry as well as its intimate connection to algebraic geometry. It is one of the few highlights in Kähler geometry which deserve new proofs by the Ricci flow. On the other hand, the Kähler Ricci flow is a natural way to understand the Calabi conjecture in the Fano setting. Following Yau's estimate ([Yau78]), H. D. Cao [Cao85] proved that the Kähler Ricci flow with smooth initial metric always exists globally. On a KE manifold, the first named author and Tian showed that the Kähler Ricci flow converges exponentially fast toward the KE metric if the initial metric has positive bisectional curvature (cf. [CT1], [CT2]). Using his famous $\mu$-functional, Perelman proved that scalar curvature, diameter and normalized Ricci potentials are all uniformly bounded along the Kähler Ricci flow (cf. [SeT]). These fundamental estimates of G. Perelman opened the door for a more qualitative analysis of singularities formed in the Kähler Ricci flow. As a corollary of his estimates, G. Perelman announced that the Kähler Ricci flow always converges to the KE metric on every KE manifold. The first written proof of this statement appeared in [TZ] where Tian and Zhu also generalized it to Kähler manifolds with KRS (Kähler Ricci soliton) metrics. In our humble view, the estimates of G. Perelman make the flow approach a plausible one in terms of understanding the Calabi conjecture in the Fano setting. We hope that this modest progress in the Kähler Ricci flow will attract more attention to the renown Hamilton-Tian conjecture that any Kähler Ricci flow converges to some KRS with mild singularities in the Cheeger-Gromov topology.

Application of the strong partial $C^{0}$-estimate is one of the crucial components of this paper. It sets up the framework of our proof for the convergence of 2-dimensional Kähler Ricci flow. This estimate originates from the strong partial $C^{0}$-estimate along continuous paths in Tian's original proof (cf. [Tian90]). He also conjectured that the strong partial $C^{0}$-estimate holds along continuous paths in higher dimensional Kähler manifolds (cf. [Tian91]).

The organization of this paper is as follows. In Section 2, along each tamed flow, we reduce the $C^{0}$-estimate of the potential function $\varphi$ to the calculation of local $\alpha$-invariants of sections $S \in H^{0}\left(K_{M}^{-v}\right)$. In Section 3, we study the fundamental properties of plurianticanonical holomorphic sections along the Kähler Ricci flow. Here we discuss applications of Hörmander's $L^{2}$-estimate of the $\bar{\partial}$-operator and we deduce uniform bounds of $|S|_{h_{t}^{v}}$ and $|\nabla S|_{h_{t}^{v}}$. Using these estimates, we obtain a theorem giving some tameness criteria. In Section 4, we calculate $\alpha_{v, k}(M)(k=1,2)$ when $c_{1}^{2}(M)=1$ or 3 and show that their values are large enough to obtain the $C^{0}$-estimate of the evolving potential function $\varphi(t)$. 


\section{Estimates along the Kähler Ricci flow}

\subsection{Fundamentals of Kähler geometry}

Let $M$ be an $n$-dimensional compact Kähler manifold. A Kähler metric can be given by its Kähler form $\omega$ on $M$. In local coordinates $z_{1}, \ldots, z_{n}, \omega$ is of the form

$$
\omega=\sqrt{-1} \sum_{i, j=1}^{n} g_{i} \bar{j} d z^{i} \wedge d z^{\bar{j}}>0
$$

where $\left\{g_{i}\right\}$ is a positive definite Hermitian matrix function. The Kähler condition requires that $\omega$ is a closed positive $(1,1)$-form. Given a Kähler metric $\omega$, the curvature tensor is

$$
R_{i \bar{j} k \bar{l}}=-\frac{\partial^{2} g_{i \bar{j}}}{\partial z^{k} \partial z^{\bar{l}}}+\sum_{p, q=1}^{n} g^{p \bar{q}} \frac{\partial g_{i \bar{q}}}{\partial z^{k}} \frac{\partial g_{p \bar{j}}}{\partial z^{\bar{l}}}, \quad \forall i, j, k, l=1, \ldots, n .
$$

The Ricci curvature form is

$$
\operatorname{Ric}(\omega)=\sqrt{-1} \sum_{i, j=1}^{n} R_{i}(\omega) d z^{i} \wedge d z^{\bar{j}}=-\sqrt{-1} \partial \bar{\partial} \log \operatorname{det}\left(g_{k \bar{l}}\right)
$$

It is a real and closed $(1,1)$-form. Note that $[\mathrm{Ric}]=2 \pi c_{1}(M)$.

Now we assume that $M$ is Fano, i.e., $c_{1}(M)>0$. Fix an initial metric $\omega=\omega_{0}$ in the class $2 \pi c_{1}(M)$. The normalized Ricci flow equation (cf. [Cao85]) is

$$
\frac{\partial g_{i \bar{j}}}{\partial t}=g_{i \bar{j}}-R_{i \bar{j}}, \quad \forall i, j=1, \ldots, n .
$$

It is easy to check that this flow preserves the class $2 \pi c_{1}(M)$. Therefore, every metric form $\omega_{t}$ can be written as $\omega_{\varphi(t)}=\omega+\sqrt{-1} \partial \bar{\partial} \varphi(t)$ where $\varphi(t)$ is the Kähler potential. On the level of Kähler potentials, the flow becomes

$$
\frac{\partial \varphi}{\partial t}=\log \frac{\omega_{\varphi}^{n}}{\omega^{n}}+\varphi+u_{\omega}
$$

where $u_{\omega}$ is defined by

$$
\operatorname{Ric}(\omega)-\omega=-\sqrt{-1} \partial \bar{\partial} u_{\omega}, \quad \text { and } \quad \int_{X}\left(e^{-u_{\omega}}-1\right) \omega^{n}=0 .
$$

The flow equation (2) or (3) is referred to as the Kähler Ricci flow on M. It was proved by Cao [Cao85], who followed Yau's celebrated work [Yau78], that the Kähler Ricci flow exists globally for any smooth initial Kähler metric in $2 \pi c_{1}(M)$.

For the given $\omega \in 2 \pi c_{1}(M)$, define $\mathscr{P}(M, \omega)=\{\varphi \mid \omega+\sqrt{-1} \partial \bar{\partial} \varphi>0\}$. It is shown in [Tian87] that there is a small constant $\delta>0$ such that

$$
\sup _{\varphi \in \mathscr{P}(M, \omega)} \frac{1}{V} \int_{M} e^{-\delta\left(\varphi-\sup _{M} \varphi\right)} \omega^{n}<\infty .
$$


The supremum of such $\delta$ 's depends only on the class $[\omega]=2 \pi c_{1}(M)$. It is called the $\alpha$-invariant of $M$ and denoted by $\alpha(M)$. If $G$ is a compact subgroup of $\operatorname{Aut}(M)$ and $\omega$ is a $G$-invariant metric form, we define

$$
\mathscr{P}_{G}(M, \omega)=\{\varphi \mid \omega+\sqrt{-1} \partial \bar{\partial} \varphi>0, \varphi \text { is invariant under } G\} .
$$

Similarly, we can define $\alpha_{G}(M)$. Actually, $\alpha_{G}(M)$ is an algebraic invariant. It is called the global $\log$ canonical threshold $\operatorname{lct}(X, G)$ by algebraic geometers. See [Chl] for more details.

\subsection{Known estimates along general Kähler Ricci flow}

There are a lot of estimates along the Kähler Ricci flow in the literature. We list some of them which are important to our arguments.

Proposition 2.1 (Perelman, cf. [SeT]). Suppose $\left\{\left(M^{n}, g(t)\right), 0 \leq t<\infty\right\}$ is a Kähler Ricci flow solution. There are two positive constants $\mathcal{B}, \kappa$ depending only on this flow such that the following two estimates hold.

1. Suppose $-u_{t}$ is the normalized Ricci potential of $\omega_{t}$, i.e.,

$$
\operatorname{Ric}\left(\omega_{t}\right)-\omega_{t}=-\sqrt{-1} \partial \bar{\partial} u_{t}, \quad \frac{1}{V} \int_{M} e^{-u_{t}} \omega_{t}^{n}=1 .
$$

Then

$$
\left\|R\left(\omega_{t}\right)\right\|_{C^{0}}+\operatorname{diam}_{g(t)} M+\left\|u_{t}\right\|_{C^{0}}+\left\|\nabla u_{t}\right\|_{C^{0}}<\mathcal{B}
$$

uniformly for every $t \in[0, \infty)$.

2. Under the metric $g(t)$, $\operatorname{Vol}(B(x, r)) / r^{2 n}>\kappa$ for every $r \in(0,1),(x, t) \in M \times[0, \infty)$.

After this fundamental work of G. Perelman, many interesting papers appeared. We include a few references here for the convenience of the readers: [CH], [CST], [CW1], [CW2], [Hei], [PSS], [PSSW1], [PSSW2], [Ru], [RZZ], [Se1], [Se2], [TZs], etc. In this subsection, we cite a few results which are directly related to our work here.

Proposition 2.2 ([Zhq], [Ye]). There is a uniform Sobolev constant $C_{S}$ along the Kähler Ricci flow solution $\left\{\left(M^{n}, g(t)\right), 0 \leq t<\infty\right\}$. In other words, for every $f \in C^{\infty}(M)$, we have

$$
\left(\int_{M}|f|^{\frac{2 n}{n-1}} \omega_{t}^{n}\right)^{\frac{n-1}{n}} \leq C_{S}\left\{\int_{M}|\nabla f|^{2} \omega_{t}^{n}+\frac{1}{V^{1 / n}} \int_{M}|f|^{2} \omega_{t}^{n}\right\} .
$$

As an easy application of a normalization technique initiated in [CT1], one can prove the following property.

Proposition 2.3 (cf. [PSS]). By properly choosing the initial condition, we have

$$
\|\dot{\varphi}\|_{C^{0}}+\|\nabla \dot{\varphi}\|_{C^{0}}<C
$$

for some constant $C$ independent of time $t$. 
Based on these estimates, the authors proved the following properties.

Proposition 2.4 (cf. [Ru], [CW2]). There is a constant $C$ such that

$$
\frac{1}{V} \int_{M}(-\varphi) \omega_{\varphi}^{n} \leq \sup _{M} \varphi-\sum_{i=0}^{n-1} \frac{i}{V} \int_{M} \sqrt{-1} \partial \varphi \wedge \bar{\partial} \varphi \wedge \omega^{i} \wedge \omega_{\varphi}^{n-1-i}+C
$$

In particular,

$$
\frac{1}{V} \int_{M}(-\varphi) \omega_{\varphi}^{n} \leq n \sup _{M} \varphi+C
$$

Proposition 2.5 (cf. [Ru], [CW2]). For every $\delta$ less than the $\alpha$-invariant of $M$, there is a uniform constant $C$ such that

$$
\sup _{M} \varphi<\frac{1-\delta}{\delta} \int_{M}(-\varphi) \omega_{\varphi}^{n}+C
$$

along the flow.

Lemma 2.1 (cf. [Ru], [CW2]). Along the Kähler Ricci flow $\left\{\left(M^{n}, g(t)\right), 0 \leq t<\infty\right\}$ on the Fano manifold $M$, the following conditions are equivalent:

- $\varphi$ is uniformly bounded.

- $\sup _{M} \varphi$ is uniformly bounded from above.

- $\inf _{M} \varphi$ is uniformly bounded from below.

- $\int_{M} \varphi \omega^{n}$ is uniformly bounded from above.

- $\int_{M}(-\varphi) \omega_{\varphi}^{n}$ is uniformly bounded from above.

- $I_{\omega}(\varphi)$ is uniformly bounded.

- $\operatorname{Osc}_{M} \varphi$ is uniformly bounded.

Here $I_{\omega}(\varphi)=\frac{1}{\operatorname{Vol}(M)} \int_{M} \varphi\left(\omega^{n}-\omega_{\varphi}^{n}\right)$.

As a simple corollary, we have

Theorem 2.1 (cf. [Ru], [CW2]). If $\alpha_{G}(M)>n /(n+1)$, then $\varphi$ is uniformly bounded along the Kähler Ricci flow initiating from some $G$-invariant metric $\omega \in 2 \pi c_{1}(M)$.

\subsection{Estimates along the tamed Kähler Ricci flow}

In this section, we only study the tamed Kähler Ricci flow.

Definition 2.1. For every positive integer $v$, we can define a function $F_{v}$ on $M \times[0, \infty)$ as follows:

$$
F_{v}(x, t) \triangleq \frac{1}{v} \log \sum_{\beta=0}^{N_{v}}\left|S_{v, \beta}^{t}\right|_{h_{t}^{v}}^{2}(x)
$$


where $\left\{S_{\nu, \beta}^{t}\right\}_{\beta=0}^{N_{v}}$ is an orthonormal basis of $H^{0}\left(K_{M}^{-\nu}\right)$ under the metrics $g_{t}=g(t)$ and $h_{t}^{v}=\left(\operatorname{det} g_{t}\right)^{\nu}$, i.e.,

$$
\int_{M}\left\langle S_{v, \alpha}^{t}, S_{v, \beta}^{t}\right\rangle_{h_{t}^{\nu}} \omega_{t}^{n}=\delta_{\alpha \beta}, \quad N_{\nu}=\operatorname{dim} H^{0}\left(K_{M}^{-\nu}\right)-1 .
$$

Note that this definition is independent of the choice of the orthonormal basis of $H^{0}\left(K_{M}^{-\nu}\right)$.

Definition 2.2. $\left\{\left(M^{n}, g(t)\right), 0 \leq t<\infty\right\}$ is called a flow tamed by $v$ if:

- $K_{M}^{-v}$ is very ample.

- $\left|F_{v}\right|_{C^{0}(M \times[0, \infty))}<\infty$.

A flow $\left\{\left(M^{n}, g(t)\right), 0 \leq t<\infty\right\}$ is called a tamed flow if it is tamed by some large integer $v$.

Suppose $\left\{\left(M^{n}, g(t)\right), 0 \leq t<\infty\right\}$ is a Kähler Ricci flow tamed by $v$. For simplicity of notation, denote $N_{v}$ by $N$. Under the metrics $g_{t}$ and $h_{t}^{v}$, we choose $\left\{S_{v, \beta}^{t}\right\}_{\beta=0}^{N}$ as an orthonormal basis of $H^{0}\left(K_{M}^{-\nu}\right)$. At the same time, let $\left\{\tilde{S}_{\nu, \beta}^{t}\right\}_{\beta=0}^{N}$ be an orthonormal basis of $H^{0}\left(K_{M}^{-\nu}\right)$ under the metrics $g_{0}$ and $h_{0}^{\nu}$. Then we have two embeddings

$$
\begin{aligned}
\Phi^{t}: M \rightarrow \mathbb{C P}^{N}, & x \mapsto\left[S_{v, 0}^{t}(x): \cdots: S_{v, N}^{t}(x)\right] ; \\
\Psi^{t}: M \rightarrow \mathbb{C P}^{N}, & x \mapsto\left[\tilde{S}_{v, 0}^{t}(x): \cdots: \tilde{S}_{v, N}^{t}(x)\right] .
\end{aligned}
$$

By rotating the basis if necessary, we can assume $\Phi^{t}=\sigma(t) \circ \Psi^{t}$ where

$\sigma(t)=a(t) \operatorname{diag}\left\{\lambda_{0}(t), \ldots, \lambda_{N}(t)\right\}, a(t)>0,0<\lambda_{0}(t)<\lambda_{1}(t)<\cdots<\lambda_{N}(t)=1$.

This indicates that the Kähler Ricci flow equation can be rewritten as

$$
\begin{aligned}
\dot{\varphi} & =\log \frac{\omega_{\varphi}^{n}}{\omega^{n}}+\varphi+u_{\omega}=\frac{1}{v} \log \frac{\sum_{\beta=0}^{N}\left|S_{v, \beta}^{t}\right|_{h_{t}^{v}}^{2}}{\sum_{\beta=0}^{N}\left|S_{v, \beta}^{t}\right|_{h_{0}^{v}}^{2}}+\varphi+u_{\omega} \\
& =\frac{1}{v} \log \sum_{\beta=0}^{N}\left|S_{v, \beta}^{t}\right|_{h_{t}^{v}}^{2}-\frac{1}{v} \log \sum_{\beta=0}^{N}\left|a(t) \lambda_{\beta}(t) \tilde{S}_{v, \beta}^{t}\right|_{h_{0}^{v}}^{2}+\varphi+u_{\omega} \\
& =F_{\nu}(x, t)-\frac{1}{v} \log \sum_{\beta=0}^{N}\left|\lambda_{\beta}(t) \tilde{S}_{v, \beta}^{t}\right|_{h_{0}^{v}}^{2}+\varphi+u_{\omega}-\frac{2}{v} \log a(t) .
\end{aligned}
$$

In other words,

$$
\varphi-\frac{2}{v} \log a(t)=\dot{\varphi}-u_{\omega}-F_{v}(x, t)+\frac{1}{v} \log \sum_{\beta=0}^{N}\left|\lambda_{\beta}(t) \tilde{S}_{v, \beta}^{t}\right|_{h_{0}^{\nu}}^{2} .
$$

Since $F_{v}(x, t), \dot{\varphi}$ and $u_{\omega}$ are all uniformly bounded, we obtain

$$
\varphi-\frac{2}{v} \log a(t) \sim \frac{1}{v} \log \sum_{\beta=0}^{N}\left|\lambda_{\beta}(t) \tilde{S}_{v, \beta}^{t}\right|_{h_{0}^{v}}^{2}
$$


Here we use $\sim$ to denote that the difference of the two sides is controlled by a constant. It follows that

$$
\varphi-\sup _{M} \varphi \sim \frac{1}{v} \log \sum_{\beta=0}^{N}\left|\lambda_{\beta}(t) \tilde{S}_{v, \beta}^{t}\right|_{h_{0}^{v}}^{2}-\frac{1}{v} \sup _{M} \log \sum_{\beta=0}^{N}\left|\lambda_{\beta}(t) \tilde{S}_{v, \beta}^{t}\right|_{h_{0}^{v}}^{2} .
$$

It is obvious that

$$
\sup _{M} \log \sum_{\beta=0}^{N}\left|\lambda_{\beta}(t) \tilde{S}_{\nu, \beta}^{t}\right|_{h_{0}^{v}}^{2} \leq \sup _{M} \log \sum_{\beta=0}^{N}\left|\tilde{S}_{\nu, \beta}^{t}\right|_{h_{0}^{v}}^{2}<C .
$$

On the other hand, we have

$$
\begin{aligned}
\sup _{M} \log \sum_{\beta=0}^{N}\left|\lambda_{\beta}(t) \tilde{S}_{v, \beta}^{t}\right|_{h_{0}^{v}}^{2} & \geq \sup _{M} \log \left|\lambda_{N}(t) \tilde{S}_{N, \beta}^{t}\right|_{h_{0}^{v}}^{2}=\sup _{M} \log \left|\tilde{S}_{v, N}^{t}\right|_{h_{0}^{v}}^{2} \\
& =\log \sup _{M}\left|\tilde{S}_{v, N}^{t}\right|_{h_{0}^{v}}^{2} \geq \log \frac{1}{V} \int_{M}\left|\tilde{S}_{v, N}^{t}\right|_{h_{0}^{v}}^{2} \omega^{n}=-\log V .
\end{aligned}
$$

Therefore, $v^{-1} \sup _{M} \log \sum_{\beta=0}^{N}\left|\lambda_{\beta}(t) \tilde{S}_{v, \beta}^{t}\right|_{h_{0}^{v}}^{2}$ is uniformly bounded, which yields

$$
\varphi-\sup _{M} \varphi \sim \frac{1}{v} \log \sum_{\beta=0}^{N}\left|\lambda_{\beta}(t) \tilde{S}_{v, \beta}^{t}\right|_{h_{0}^{v}}^{2}
$$

So we have proved the following property.

Proposition 2.6. If $\left\{\left(M^{n}, g(t)\right), 0 \leq t<\infty\right\}$ is a Kähler Ricci flow tamed by $v$, then there is a constant $C$ (depending on this flow and $v)$ such that

$$
\left.\left|\varphi-\sup _{M} \varphi-\frac{1}{v} \log \sum_{\beta=0}^{N}\right| \lambda_{\beta}(t) \tilde{S}_{v, \beta}^{t}\right|_{h_{0}^{v}} ^{2} \mid<C
$$

uniformly along this flow.

Inequality (8) is called the strong partial $C^{0}$-estimate by Tian. Using this estimate, the control of $\|\varphi\|_{C^{0}(M)}$ along the Kähler Ricci flow is reduced to the control of values of local $\alpha$-invariants (see Definitions 1 and 2) of holomorphic sections $S \in H^{0}\left(K_{M}^{-v}\right)$.

Theorem 2.2. Suppose $\left\{\left(M^{n}, g(t)\right), 0 \leq t<\infty\right\}$ is a Kähler Ricci flow tamed by $v$. If $\alpha_{v, 1}>n /(n+1)$, then $\varphi$ is uniformly bounded along this flow. In particular, this flow converges to a KE metric exponentially fast. 
Proof. Suppose not. Then there is a sequence of times $t_{i}$ such that $\lim _{i \rightarrow \infty}\left|\varphi_{t_{i}}\right|_{C^{0}(M)}$ $=\infty$.

Choose $S_{v, \beta}^{t}=a(t) \lambda_{\beta}(t) \tilde{S}_{v, \beta}^{t}, 0 \leq \beta \leq N$, as before. Since both $\left|\tilde{S}_{v, \beta}^{t}\right|_{h_{0}^{v}}$ and $\lambda_{\beta}(t)$ are uniformly bounded, we can assume

$$
\lim _{i \rightarrow \infty} \lambda_{\beta}\left(t_{i}\right)=\bar{\lambda}_{\beta}, \quad \lim _{i \rightarrow \infty} \tilde{S}_{v, \beta}^{t_{i}}=\bar{S}_{v, \beta}, \quad \beta=0,1, \ldots, N .
$$

Notice that $\bar{\lambda}_{N}=1$.

Define

$$
I(\alpha, t)=\int_{M}\left(\sum_{\beta=0}^{N}\left|\lambda_{\beta}(t) \tilde{S}_{v, \beta}^{t}\right|_{h_{0}^{v}}^{2}\right)^{-\alpha / v} \omega^{n} .
$$

Clearly, $I\left(\alpha, t_{i}\right) \leq \int_{M}\left|\tilde{S}_{v, N}^{t_{i}}\right|_{h_{0}^{v}}^{-2 \alpha / v} \omega^{n}$. As $\bar{S}_{v, N} \in H^{0}\left(K_{M}^{-v}\right)$ and $\alpha_{v, 1}>n /(n+1)$, we can find a number $\alpha \in\left(n /(n+1), \alpha_{\nu, 1}\right)$ such that $\int_{M}\left|\bar{S}_{\nu, N}\right|_{h_{0}^{\nu}}^{-2 \alpha / v} \omega^{n}<C$. By the semicontinuity of the singularity exponent (cf. [Tian90], [DK], [PS]), we have

$$
\limsup _{i \rightarrow \infty} I\left(\alpha, t_{i}\right) \leq \lim _{i \rightarrow \infty} \int_{M}\left|\tilde{S}_{v, N}^{t_{i}}\right|_{h_{0}^{v}}^{-2 \alpha / v} \omega^{n}=\int_{M}\left|\bar{S}_{v, N}\right|_{h_{0}^{v}}^{-2 \alpha / v} \omega^{n}<C .
$$

Along a tamed flow, the inequality

$$
\left.\left|\varphi-\sup _{M} \varphi-\frac{1}{v} \log \sum_{\beta=0}^{N}\right| \lambda_{\beta}(t) \tilde{S}_{v, \beta}^{t}\right|_{h_{0}^{v}} ^{2} \mid<C
$$

holds. It follows that $\int_{M} e^{\alpha\left(\varphi_{t_{i}}-\sup _{M} \varphi_{t_{i}}\right)} \omega^{n}<C$. Recalling that $\dot{\varphi}=\log \left(\omega_{\varphi}^{n} / \omega^{n}\right)+\varphi+u_{\omega}$, we have

$$
\frac{1}{V} \int_{M} e^{-\alpha\left(\varphi_{t_{i}}-\sup _{M} \varphi_{t_{i}}\right)} \cdot e^{\varphi_{t_{i}}+u_{\omega}-\dot{\varphi}} \omega_{t_{i}}^{n}<C .
$$

Note that both $\dot{\varphi}$ and $u_{\omega}$ are uniformly bounded. It follows from the convexity of the exponential map that $\alpha \sup _{M} \varphi_{t_{i}}+(1-\alpha) \frac{1}{V} \int_{M} \varphi_{t_{i}} \omega_{t_{i}}^{n}<C$. In other words,

$$
\sup _{M} \varphi_{t_{i}}<\frac{1-\alpha}{\alpha} \frac{1}{V} \int_{M}\left(-\varphi_{t_{i}}\right) \omega_{t_{i}}^{n}+C
$$

Combining this with inequality (5), we have

$$
\sup _{M} \varphi_{t_{i}}<n \frac{1-\alpha}{\alpha} \sup _{M} \varphi_{t_{i}}+C .
$$

Since $\alpha \in(n /(n+1), 1)$, it follows that $\sup _{M} \varphi_{t_{i}}$ is uniformly bounded from above. Consequently, $\varphi_{t_{i}}$ is uniformly bounded. This contradicts our assumption on $\varphi_{t_{i}}$.

By a more careful analysis, we can improve this theorem a little bit. 
Proposition 2.7. Let $X_{t} \triangleq v^{-1} \log \left(\sum_{\beta=0}^{N}\left|\lambda_{\beta}(t) \tilde{S}_{v, \beta}^{t}\right|_{h_{0}^{v}}^{2}\right)$. There is a constant $C$ such that

$$
\left|\frac{1}{V} \int_{M}\left\{\sqrt{-1} \partial \varphi_{t_{i}} \wedge \bar{\partial} \varphi_{t_{i}}-\sqrt{-1} \partial X_{t_{i}} \wedge \bar{\partial} X_{t_{i}}\right\} \wedge \omega^{n-1}\right|<C .
$$

Proof. Since every $\tilde{S}_{v, \beta}^{t_{i}}$ is a holomorphic section, direct calculation shows that

$$
\Delta X_{t_{i}} \geq-R
$$

where $\Delta, R$ are the Laplacian operator and the scalar curvature under the metric $\omega$. As $\Delta \varphi+n>0$, we can choose a constant $C_{0}$ such that $\Delta \varphi+\Delta X_{t_{i}}+C_{0}>0$. For the simplicity of notation, we omit the subscript $t_{i}$ in the following arguments. So

$$
\left|\varphi-\sup _{M} \varphi-X\right|<C, \quad \Delta X+\Delta \varphi+C_{0}>0
$$

Having these conditions at hand, direct computation gives

$$
\begin{aligned}
& \frac{1}{V} \int_{M}\{\sqrt{-1} \partial \varphi \wedge \bar{\partial} \varphi-\sqrt{-1} \partial X \wedge \bar{\partial} X\} \wedge \omega^{n-1}=\frac{1}{V} \int_{M}(X-\varphi)(\Delta X+\Delta \varphi) \omega^{n} \\
& =\frac{1}{V} \int_{M}\left(X-\varphi+\sup _{M} \varphi+C\right)(\Delta X+\Delta \varphi) \omega^{n} \\
& =\frac{1}{V} \int_{M}\left(X-\varphi+\sup _{M} \varphi+C\right)\left(\Delta X+\Delta \varphi+C_{0}\right) \omega^{n}-\frac{C_{0}}{V} \int_{M}\left(X-\varphi+\sup _{M} \varphi+C\right) \omega^{n} \\
& \geq-\frac{C_{0}}{V} \int_{M}\left(X-\varphi+\sup _{M} \varphi+C\right) \omega^{n} \geq-2 C_{0} C .
\end{aligned}
$$

On the other hand, a similar calculation shows

$$
\begin{aligned}
\frac{1}{V} \int_{M}\{\sqrt{-1} \partial \varphi \wedge \bar{\partial} \varphi-\sqrt{-1} \partial X \wedge \bar{\partial} X\} \wedge \omega^{n-1} & \leq-\frac{C_{0}}{V} \int_{M}\left(X-\varphi+\sup _{M} \varphi-C\right) \omega^{n} \\
& \leq 2 C_{0} C .
\end{aligned}
$$

Consequently,

$$
\left|\frac{1}{V} \int_{M}\{\sqrt{-1} \partial \varphi \wedge \bar{\partial} \varphi-\sqrt{-1} \partial X \wedge \bar{\partial} X\} \wedge \omega^{n-1}\right| \leq 2 C_{0} C .
$$

It follows from this proposition that inequality (4) implies

$$
\frac{1}{V} \int_{M}(-\varphi) \omega_{\varphi}^{n} \leq n \sup _{M} \varphi-\frac{n-1}{V} \int_{M} \sqrt{-1} \partial X \wedge \bar{\partial} X \wedge \omega^{n-1}+C .
$$

Similar to the theorems in [Tian90], we can prove the following theorem. 
Theorem 2.3. Suppose $\left\{\left(M^{n}, g(t)\right), 0 \leq t<\infty\right\}$ is a Kähler Ricci flow tamed by $v$, and $M$ is a Fano manifold satisfying

$$
\alpha_{\nu, 2}>\frac{n}{n+1} \quad \text { and } \quad \alpha_{\nu, 1}>\frac{1}{2-\frac{n-1}{(n+1) \alpha_{\nu, 2}}} .
$$

Then along this flow, $\varphi$ is uniformly bounded. In particular, this flow converges to a KE metric exponentially fast.

Proof. Suppose not. We have a sequence of times $t_{i}$ such that $\lim _{i \rightarrow \infty}\left|\varphi_{t_{i}}\right|_{C^{0}(M)}=\infty$. As before, we have

$$
\lim _{i \rightarrow \infty} \tilde{S}_{v, \beta}^{t_{i}}=\bar{S}_{v, \beta}, \quad \beta=0,1, \ldots, N ; \quad \lim _{i \rightarrow \infty} \lambda_{\beta}\left(t_{i}\right)=\bar{\lambda}_{\beta} ; \quad \bar{\lambda}_{N}=1 .
$$

Claim 1. $\bar{\lambda}_{N-1}=0$.

Otherwise, $\bar{\lambda}_{N-1}>0$. Fixing some $\alpha \in\left(n /(n+1), \alpha_{v, 2}\right)$, we calculate

$$
\begin{aligned}
I\left(\alpha, t_{i}\right) & =\int_{M}\left(\sum_{\beta=0}^{N}\left|\lambda_{\beta}(t) \tilde{S}_{v, \beta}^{t_{i}}\right|_{h_{0}^{v}}^{2}\right)^{-\alpha / v} \omega^{n} \leq \int_{M}\left(\left|\lambda_{N-1}\left(t_{i}\right) \tilde{S}_{v, N-1}^{t_{i}}\right|_{h_{0}^{v}}^{2}+\left|\tilde{S}_{v, N}^{t_{i}}\right|_{h_{0}^{v}}^{2}\right)^{-\alpha / v} \omega^{n} \\
& \leq\left(\lambda_{N-1}\left(t_{i}\right)\right)^{-2 \alpha} \int_{M}\left(\left|\tilde{S}_{v, N-1}^{t_{i}}\right|_{h_{0}^{v}}^{2}+\left|\tilde{S}_{v, N}^{t_{i}}\right|_{h_{0}^{v}}^{2}\right)^{-\alpha / v} \omega^{n} .
\end{aligned}
$$

For simplicity of notation, we consider $h_{0}^{v}$ as the default metric on the line bundle $K_{M}^{-v}$ without writing it out explicitly. Then the semicontinuity property implies

$$
\lim _{i \rightarrow \infty} \int_{M}\left(\left|\tilde{S}_{v, N-1}^{t_{i}}\right|^{2}+\left|\tilde{S}_{v, N}^{t_{i}}\right|^{2}\right)^{-\alpha / v} \omega^{n}=\int_{M}\left(\left|\bar{S}_{v, N-1}\right|^{2}+\left|\bar{S}_{v, N}\right|^{2}\right)^{-\alpha / v} \omega^{n}<\infty .
$$

It follows that

$$
I\left(\alpha, t_{i}\right)<2\left(\bar{\lambda}_{N-1}\right)^{-2 \alpha / \nu} \int_{M}\left(\left|\bar{S}_{\nu, N-1}\right|^{2}+\left|\bar{S}_{v, N}\right|^{2}\right)^{-\alpha / v} \omega^{n}<C_{\alpha} .
$$

Recalling the definition of $I\left(\alpha, t_{i}\right)$, inequality (8) implies $\int_{M} e^{-\alpha\left(\varphi\left(t_{i}\right)-\sup _{M} \varphi\left(t_{i}\right)\right)} \omega^{n}<C$. Since $\alpha>n /(n+1)$, as in the previous theorem we obtain $\left|\varphi_{t_{i}}\right|_{C^{0}(M)}<C$. This contradicts the initial assumption on $\varphi_{t_{i}}$. Therefore $\bar{\lambda}_{N-1}=0$ as claimed.

Claim 2. For every small constant $\epsilon$, there is a constant $C$ such that

$$
\begin{aligned}
(1-\epsilon) \alpha_{\nu, 2} \sup _{M} \varphi_{t_{i}}+\left(1-(1-\epsilon) \alpha_{\nu, 2}\right) & \frac{1}{V} \int_{M} \varphi_{t_{i}} \omega_{t_{i}}^{n} \\
& \leq-2(1-\epsilon) \frac{\alpha_{v, 2}}{v} \log \lambda_{N-1}\left(t_{i}\right)+C .
\end{aligned}
$$


Fixing $\epsilon$ small, we have

$$
\begin{aligned}
I\left((1-\epsilon) \alpha_{v, 2}, t_{i}\right) & =\int_{M}\left(\sum_{\beta=0}^{N}\left|\lambda_{\beta} \tilde{S}_{v, \beta}^{t_{i}}\right|^{2}\right)^{-(1-\epsilon) \alpha_{v, 2} / v} \omega^{n} \\
& \leq \int_{M}\left\{\lambda_{N-1}\left(t_{i}\right)^{2}\left(\left|\tilde{S}_{v, N-1}^{t_{i}}\right|^{2}+\left|\tilde{S}_{v, N}^{t_{i}}\right|^{2}\right)\right\}^{-(1-\epsilon) \alpha_{v, 2} / v} \omega^{n} \\
& <C \lambda_{N-1}\left(t_{i}\right)^{-2(1-\epsilon) \alpha_{v, 2} / \nu} .
\end{aligned}
$$

The tameness condition implies that

$$
\int_{M} e^{-(1-\epsilon) \alpha_{\nu, 2}\left(\varphi_{t_{i}}-\sup _{M} \varphi_{t_{i}}\right)} \omega^{n}<C \lambda_{N-1}\left(t_{i}\right)^{-2(1-\epsilon) \alpha_{\nu, 2} / \nu} .
$$

Plugging the equation $\dot{\varphi}=\log \left(\omega_{\varphi}^{n} / \omega^{n}\right)+\varphi+u_{\omega}$ into the previous inequality implies

$$
\begin{aligned}
& C \lambda_{N-1}\left(t_{i}\right)^{-2(1-\epsilon) \alpha_{v, 2} / v}>\int_{M} e^{-(1-\epsilon) \alpha_{\nu, 2}\left(\varphi_{t_{i}}-\sup _{M} \varphi_{t_{i}}\right)} \cdot e^{\varphi_{t_{i}}+u_{\omega}-\dot{\varphi}_{t_{i}}} \omega_{t_{i}}^{n} \\
& \quad=\int_{M} e^{(1-\epsilon) \alpha_{v, 2} \sup _{M} \varphi_{t_{i}}+\left(1-(1-\epsilon) \alpha_{v, 2}\right) \varphi_{t_{i}} \cdot e^{u_{\omega}-\dot{\varphi}_{t_{i}}} \omega_{t_{i}}^{n}} \\
& \geq e^{-\left\|u_{\omega}\right\|_{C^{0}(M)}-\left\|\dot{\varphi}_{t_{i}}\right\|_{C^{0}(M)}} \int_{M} e^{(1-\epsilon) \alpha_{v, 2} \sup _{M} \varphi_{t_{i}}+\left(1-(1-\epsilon) \alpha_{v, 2}\right) \varphi_{t_{i}}} \omega_{t_{i}}^{n} \\
& \geq e^{-\left\|u_{\omega}\right\|_{C^{0}(M)}-\left\|\dot{\varphi}_{t_{i}}\right\|_{C^{0}(M)}} \cdot V \cdot e^{(1-\epsilon) \alpha_{v, 2} \sup _{M} \varphi_{t_{i}}+\left(1-(1-\epsilon) \alpha_{v, 2}\right) \frac{1}{V} \int_{M} \varphi_{t_{i}} \omega_{t_{i}}^{n}}
\end{aligned}
$$

Taking logarithms on both sides, we obtain (12). This finishes the proof of Claim 2.

Claim 3. For every small number $\epsilon>0$, there is a constant $C_{\epsilon}$ such that

$$
\frac{1}{V} \int_{M} \sqrt{-1} \partial X_{t_{i}} \wedge \bar{\partial} X_{t_{i}} \wedge \omega^{n-1} \geq-\frac{1-\epsilon}{\nu} \log \lambda_{N-1}\left(t_{i}\right)-C_{\epsilon} .
$$

The proof is the same as the corresponding proof in [Tian91], so we omit it.

Plugging (13) into (11), together with (12), we obtain

$$
\left\{\begin{array}{l}
\frac{1}{V} \int_{M}\left(-\varphi_{t_{i}}\right) \omega_{t_{i}}^{n} \leq n \sup _{M} \varphi_{t_{i}}+\frac{(n-1)(1-\epsilon)}{v} \log \lambda_{N-1}\left(t_{i}\right)+C, \\
(1-\epsilon) \alpha_{v, 2} \sup _{M} \varphi_{t_{i}}+\left(1-(1-\epsilon) \alpha_{v, 2}\right) \frac{1}{V} \int_{M} \varphi_{t_{i}} \omega_{t_{i}}^{n} \leq-\frac{2(1-\epsilon) \alpha_{\nu, 2}}{v} \log \lambda_{N-1}\left(t_{i}\right)+C .
\end{array}\right.
$$

Eliminating $\log \lambda_{N-1}\left(t_{i}\right)$, we have

$$
\frac{1}{V} \int_{M}\left(-\varphi_{t_{i}}\right) \omega_{t_{i}}^{n} \leq \frac{(n+1)+(n-1) \epsilon}{((n+1)-(n-1) \epsilon) \alpha_{\nu, 2}-(n-1)} \alpha_{\nu, 2} \sup _{M} \varphi_{t_{i}}+C .
$$


From inequality (9), we have $\sup _{M} \varphi_{t_{i}} \leq \frac{1-(1-\epsilon) \alpha_{\nu, 1}}{(1-\epsilon) \alpha_{\nu, 1}} \frac{1}{V} \int_{M}\left(-\varphi_{t_{i}}\right) \omega_{t_{i}}^{n}+C$. It follows that $\left\{1-\frac{(n+1)+(n-1) \epsilon}{((n+1)-(n-1) \epsilon) \alpha_{\nu, 2}-(n-1)} \cdot \alpha_{\nu, 2} \cdot \frac{1-(1-\epsilon) \alpha_{\nu, 1}}{(1-\epsilon) \alpha_{\nu, 1}}\right\} \frac{1}{V} \int_{M}\left(-\varphi_{t_{i}}\right) \omega_{t_{i}}^{n} \leq C$ for every small constant $\epsilon$ and some large constant $C$ depending on $\epsilon$. Since

$$
\alpha_{v, 1}>\frac{1}{2-\frac{n-1}{(n+1) \alpha_{v, 2}}}=\frac{A}{A+1} \quad \text { where } \quad A=\frac{(n+1) \alpha_{v, 2}}{(n+1) \alpha_{v, 2}-(n-1)},
$$

we can choose $\epsilon$ small enough such that

$$
1-\frac{(n+1)+(n-1) \epsilon}{((n+1)-(n-1) \epsilon) \alpha_{\nu, 2}-(n-1)} \cdot \alpha_{\nu, 2} \cdot \frac{1-(1-\epsilon) \alpha_{\nu, 1}}{(1-\epsilon) \alpha_{\nu, 1}}>0 .
$$

This implies that $\frac{1}{V} \int_{M}\left(-\varphi_{t_{i}}\right) \omega_{t_{i}}^{n}$ is uniformly bounded. Therefore, $\left|\varphi_{t_{i}}\right|_{C^{0}(M)}$ is uniformly bounded, a contradiction.

Remark 2.1. The methods applied in Theorems 2.2 and 2.3 originate from [Tian91].

\section{Pluri-anticanonical line bundles $K_{M}^{-v}$ and the tameness condition}

In this section, we study the basic properties of normalized holomorphic sections $S \in$ $H^{0}\left(K_{M}^{-v}\right)$ under the evolving metrics $\omega_{t}$ and $h_{t}^{v}$.

\subsection{Uniform bounds of holomorphic sections of $K_{M}^{-v}$}

Let $S$ be a normalized holomorphic section of $H^{0}\left(K_{M}^{-v}\right)$, i.e., $\int_{M}|S|_{h_{t}^{v}}^{2} \omega_{t}^{n}=1$. In this section, we will show both $\left\||S|_{h_{t}^{v}}\right\|_{C^{0}}$ and $\left\||\nabla S|_{h_{t}^{v}}\right\|_{C^{0}}$ are uniformly bounded.

Lemma 3.1. Suppose $\left\{\left(M^{n}, g(t)\right), 0 \leq t<\infty\right\}$ is a Kähler Ricci flow solution. Then there is a constant $A_{0}$ depending only on this flow such that $|S|_{h_{t}^{v}}<A_{0} v^{n / 2}$ whenever $S \in H^{0}\left(K_{M}^{-v}\right)$ satisfies $\int_{M}|S|_{h_{t}^{\nu}}^{2} \omega_{t}^{n}=1$.

Proof. Fix a time $t$ and do all the calculations under the metrics $g_{t}$ and $h_{t}^{v}$. Recall we have a uniform Sobolev constant, so we can do analysis uniformly independent of time $t$.

Claim. S satisfies the equation

$$
\Delta|S|^{2}=|\nabla S|^{2}-v R|S|^{2}
$$


This calculation can be done locally. Fix a point $x \in M$. Let $U$ be a neighborhood of $x$ with coordinates $\left\{z^{1}, \ldots, z^{n}\right\}$. Then $K_{M}^{-v}$ has a natural trivialization on the domain $U$ and we can write $S=f\left(\frac{\partial}{\partial z^{1}} \wedge \cdots \wedge \frac{\partial}{\partial z^{n}}\right)^{v}$ for some holomorphic function $f$ locally. For convenience, we denote $h=\operatorname{det} g_{k l}$. Therefore, direct calculation shows

$$
\begin{aligned}
& \Delta|S|^{2}=g^{i \bar{j}}\left\{f \bar{f} h^{\nu}\right\}_{i \bar{j}}=g^{i} \bar{j}\left\{f_{i} \bar{f} h^{\nu}+v f \bar{f} h^{\nu-1} h_{i}\right\}_{\bar{j}} \\
& =g^{i}{ }^{i}\left\{f_{i} \bar{f}_{\bar{j}} h^{v}+v \bar{f} h^{\nu-1} f_{i} h_{\bar{j}}+v f h^{\nu-1} \bar{f}_{\bar{j}} h_{i}+v(v-1) f \bar{f} h^{\nu-2} h_{i} h_{\bar{j}}+v f \bar{f} h^{\nu-1} h_{i \bar{j}}\right\} .
\end{aligned}
$$

If we choose normal coordinates at the point $x$, then we have $h=1, h_{i}=h_{\bar{j}}=0$, $h_{i \bar{j}}=-R_{i j}$. Plugging these into the previous equality we have

$$
\Delta|S|^{2}=g^{i \bar{j}}\left\{f_{i} \bar{f}_{\bar{j}}-v f \bar{f} R_{i \bar{j}}\right\}=|\nabla S|^{2}-v R|S|^{2},
$$

so equation (14) is proved.

From (14), we have

$$
\int_{M}|\nabla S|^{2} d \mu=\int_{M} \nu R|S|^{2} d \mu \leq \nu \mathcal{B}
$$

where $d \mu=\omega_{t}^{n}$. Noting that volume is fixed along the Kähler Ricci flow solution, we can omit the volume term in the Sobolev inequality by adjusting $C_{S}$. Therefore, the Sobolev inequality implies

$$
\begin{aligned}
\left\{\int_{M}|S|^{\frac{2 n}{n-1}} d \mu\right\}^{\frac{n-1}{n}} & \leq C_{S}\left\{\int_{M}|S|^{2} d \mu+\int_{M}|\nabla| S||^{2} d \mu\right\} \\
& \leq C_{S}\left\{\int_{M}|S|^{2} d \mu+\int_{M}|\nabla S|^{2} d \mu\right\} \leq C_{S}\{1+\nu \mathcal{B}\}<C \nu .
\end{aligned}
$$

Here we use the property that $\bar{\nabla} S=0$.

Note that $\Delta|S|^{2} \geq-v R|S|^{2}$. Let $u=|S|^{2}$. We have

$$
\Delta u \geq-v \mathcal{B} u, \quad\|u\|_{L^{n /(n-1)}}<C v^{1 / 2} .
$$

Multiplying this inequality by $u^{\beta-1}(\beta>1)$ and integrating by parts implies

$$
\int_{M}\left|\nabla u^{\beta / 2}\right|^{2} d \mu \leq \frac{\beta^{2}}{4(\beta-1)} \cdot(\mathcal{B} v) \cdot \int_{M} u^{\beta} d \mu .
$$

Combining this with the Sobolev inequality yields

$$
\left\{\int_{M} u^{\frac{n \beta}{n-1}} d \mu\right\}^{\frac{n-1}{n}} \leq C_{S}\left(1+\frac{\beta^{2} \mathcal{B} \nu}{4(\beta-1)}\right) \int_{M} u^{\beta} d \mu \leq C \nu \beta \int_{M} u^{\beta} d \mu .
$$

It follows that $\|u\|_{L^{n \beta /(n-1)}} \leq(C v)^{1 / \beta} \beta^{1 / \beta}\|u\|_{L^{\beta}}$. Letting $\beta=\left(\frac{n}{n-1}\right)^{k}$, we have

$$
\|u\|_{L^{\infty}} \leq(C v)^{\sum_{k=1}^{\infty}\left(\frac{n-1}{n}\right)^{k}} \cdot\left(\frac{n}{n-1}\right)^{\sum_{k=1}^{\infty} k\left(\frac{n-1}{n}\right)^{k}} \cdot\|u\|_{L^{n /(n-1)}} \leq C \nu^{\sum_{k=0}^{\infty}\left(\frac{n-1}{n}\right)^{k}}=C \nu^{n} .
$$

In other words, $\|S\|_{L^{\infty}} \leq C v^{n / 2}$. Letting $A_{0}$ be the last $C$ finishes the proof. 
Corollary 3.1. Suppose $\left\{\left(M^{n}, g(t)\right), 0 \leq t<\infty\right\}$ is a Kähler Ricci flow solution. Then

$$
F_{v}(x, t) \leq \frac{2 \log A_{0}+n \log v}{v}<B_{0}, \quad \forall x \in M, t \in[0, \infty), v \geq 1 .
$$

Here $B_{0}$ is a constant depending only on $A_{0}$.

Proof. According to the definition of $F_{\nu}$, we only need to show

$$
\sum_{\beta=0}^{N_{v}}\left|S_{\nu, \beta}^{t}\right|_{h_{t}^{v}}^{2}(x) \leq A_{0}^{2} v^{n}
$$

for every orthonormal holomorphic section basis $\left\{S_{v, \beta}^{t}\right\}_{\beta=0}^{N_{v}}$. However, by rotating the basis at the point $x$, we can always find a basis such that

$$
\left|S_{\nu, \beta}^{t}\right|_{h_{t}^{v}}^{2}(x)=0, \quad 1 \leq \beta \leq N_{\nu}
$$

Therefore, by Lemma 3.1, we have

$$
\sum_{\beta=0}^{N_{v}}\left|S_{\nu, \beta}^{t}\right|_{h_{t}^{v}}^{2}(x)=\left|S_{v, 0}^{t}\right|_{h_{t}^{v}}^{2}(x) \leq A_{0}^{2} \nu^{n}
$$

Lemma 3.2. Suppose $\left\{\left(M^{n}, g(t)\right), 0 \leq t<\infty\right\}$ is a Kähler Ricci flow solution. Then there is a constant $A_{1}$ depending only on this flow and $v$ such that $|\nabla S|_{h_{t}^{v}}<A_{1}$ whenever $S \in H^{0}\left(K_{M}^{-v}\right)$ with $\int_{M}|S|_{h_{t}^{v}}^{2} \omega_{t}^{n}=1$.

Proof. Fix a time $t$ and then do all the computations with respect to $g(t)$ and $h_{t}^{v}$. As in the previous lemma, we can do uniform analysis owing to the existence of a uniform Sobolev constant.

Claim 1. $|\nabla S|^{2}$ satisfies the equation

$$
\begin{aligned}
\Delta|\nabla S|^{2}= & |\nabla \nabla S|^{2}+v^{2}|\mathrm{Ric}|^{2}|S|^{2}-v R|\nabla S|^{2} \\
& -(2 v-1) R_{i \bar{k}} S_{k} \bar{S}_{\bar{i}}-v\left\{S R_{i} \bar{S}_{\bar{i}}+\bar{S} R_{\bar{i}} S_{i}\right\} .
\end{aligned}
$$

Locally, we can rewrite

$$
S=f\left(\frac{\partial}{\partial z^{1}} \wedge \cdots \wedge \frac{\partial}{\partial z^{n}}\right)^{v}, \quad \nabla S=\left\{f_{i}+v f(\log h)_{i}\right\} d z^{i} \otimes\left(\frac{\partial}{\partial z^{1}} \wedge \cdots \wedge \frac{\partial}{\partial z^{n}}\right)^{v},
$$

where $h=\operatorname{det} g_{k \bar{l}}$. It follows that

$$
|\nabla S|^{2}=g^{i} \bar{j}^{v}\left(f_{i}+v f(\log h)_{i}\right)\left(\bar{f}_{\bar{j}}+v \bar{f}(\log h)_{\bar{j}}\right) .
$$


Choosing normal coordinates at $x$, we have $g_{i \bar{j}}=\delta_{i \bar{j}}, h=1, h_{i}=h_{\bar{i}}=(\log h)_{i}=$ $(\log h)_{\bar{i}}=0, h_{i \bar{j}}=(\log h)_{i \bar{j}}=-R_{i \bar{j}},(\log h)_{i j}=(\log h)_{\bar{i} \bar{j}}=0$. Computation shows

$$
\begin{aligned}
\Delta|\nabla S|^{2}= & g^{k \bar{l}}\left\{g^{i \bar{j}} h^{v}\left(f_{i}+v f(\log h)_{i}\right)\left(\bar{f}_{\bar{j}}+v \bar{f}(\log h)_{\bar{j}}\right)\right\}_{k \bar{l}} \\
= & g^{k \bar{l}}\left\{-g^{i \bar{p}} g^{q \bar{j}} \frac{\partial g_{p \bar{q}}}{\partial z^{k}} h^{v}\left(f_{i}+v f(\log h)_{i}\right)\left(\bar{f}_{\bar{j}}+v \bar{f}(\log h)_{\bar{j}}\right)\right. \\
& +v g^{i \bar{j}} h^{v-1} h_{k}\left(f_{i}+v f(\log h)_{i}\right)\left(\bar{f}_{\bar{j}}+v \bar{f}(\log h)_{\bar{j}}\right) \\
& +g^{i \bar{j}} h^{v}\left(f_{i k}+v f(\log h)_{i k}+v f_{k}(\log h)_{i}\right)\left(\bar{f}_{\bar{j}}+v \bar{f}(\log h)_{\bar{j}}\right) \\
& \left.+g^{i \bar{j}} h^{v}\left(f_{i}+v f(\log h)_{i}\right) v \bar{f}(\log h)_{\bar{j} k}\right\}_{\bar{l}} \\
= & R_{j \bar{i} k \bar{k}} f_{i} \bar{f}_{\bar{j}}+v h_{k \bar{k}} f_{i} \bar{f}_{\bar{i}}+v f(\log h)_{k \bar{k} i} \bar{f}_{\bar{i}}+v f_{k}(\log h)_{i \bar{k}} \bar{f}_{\bar{i}}+f_{i k} \bar{f}_{\bar{i} \bar{k}} \\
& +v^{2} f \bar{f}_{f}(\log h)_{i \bar{k}}(\log h)_{\bar{i} k}+v f_{i} \bar{f}(\log h)_{k \bar{k} \bar{i}}+v f_{i} \bar{f}_{\bar{k}}(\log h)_{k \bar{i}} \\
= & R_{j \bar{i}} f_{i} \bar{f}_{\bar{j}}-v R|\nabla f|^{2}-v f R_{i} \bar{f}_{\bar{i}}-v R_{i \bar{k}} f_{k} \bar{f}_{\bar{i}} \\
& +|\nabla \nabla f|^{2}+v^{2}|f|^{2}|\operatorname{Ric}|^{2}-v \bar{f} f_{i} R_{\bar{i}}-v R_{i \bar{k}} f_{k} \bar{f}_{\bar{i}} \\
= & |\nabla \nabla S|^{2}+v^{2}|S|^{2}|\operatorname{Ric}|^{2}-v R|\nabla S|^{2}+(1-2 v) R_{j \bar{i}} S_{i} \bar{S}_{\bar{j}}-v\left(S R_{i} \bar{S}_{\bar{i}}+\bar{S} R_{\bar{i}} S_{i}\right) .
\end{aligned}
$$

Claim 2. S satisfies the equation

$$
S_{, i \bar{j}}=-v S R_{i \bar{j}}
$$

Locally, we can rewrite

$$
\begin{aligned}
S & =f\left(\frac{\partial}{\partial z^{1}} \wedge \cdots \wedge \frac{\partial}{\partial z^{n}}\right)^{v}, \\
\nabla S & =\left\{f_{i}+v f(\log h)_{i}\right\} d z^{i} \otimes\left(\frac{\partial}{\partial z^{1}} \wedge \cdots \wedge \frac{\partial}{\partial z^{n}}\right)^{v}, \\
\bar{\nabla} S & =f_{\bar{i}} d \bar{z}^{i} \otimes\left(\frac{\partial}{\partial z^{1}} \wedge \cdots \wedge \frac{\partial}{\partial z^{n}}\right)^{v}=0 .
\end{aligned}
$$

Recall that $\Gamma_{i j}^{k}=g^{k \bar{l}} \frac{\partial g_{i \bar{l}}}{\partial z^{j}}$ vanishes at $x$. So $(\log h)_{i},(\log h)_{i j}$ vanish at $x$. Note that $f$ is holomorphic, and our connection is compatible with both the metric and the complex structure. So $\bar{\nabla} \nabla S$ has only one term

$$
\begin{aligned}
\bar{\nabla} \nabla S & =\left\{v f(\log h)_{i \bar{j}}\right\} d \overline{z^{j}} \otimes d z^{i} \otimes\left(\frac{\partial}{\partial z^{1}} \wedge \cdots \wedge \frac{\partial}{\partial z^{n}}\right)^{v} \\
& =-v f R_{i \bar{j}} d \overline{z^{j}} \otimes d z^{i} \otimes\left(\frac{\partial}{\partial z^{1}} \wedge \cdots \wedge \frac{\partial}{\partial z^{n}}\right)^{v} .
\end{aligned}
$$

It follows that $S_{, i \bar{j}}=-v S R_{i \bar{j}}$.

Claim 3. There is a constant $C$ such that $\|\nabla S\|_{L^{2 n /(n-1)}}<C$ uniformly. 
Integrating both sides of (16) we have

$$
\begin{aligned}
\int_{M}|\nabla \nabla S|^{2} d \mu & \leq \int_{M} v R|\nabla S|^{2} d \mu+(2 v-1) \int_{M} R_{i \bar{k}} S_{k} \bar{S}_{\bar{i}} d \mu \\
& +v \int_{M}\left\{S R_{i} \bar{S}_{\bar{i}}+\bar{S} R_{\bar{i}} S_{i}\right\} d \mu
\end{aligned}
$$

where $d \mu=\omega_{t}^{n}$. Recall that $R_{i \bar{k}}=g_{i \bar{k}}-\dot{\varphi}_{i \bar{k}}$. It follows that

$$
\begin{aligned}
\int_{M}|\nabla \nabla S|^{2} d \mu \leq & \int_{M} v R|\nabla S|^{2} d \mu+(2 v-1) \int_{M}|\nabla S|^{2} d \mu-(2 v-1) \int_{M} \dot{\varphi}_{i \bar{k}} S_{k} \bar{S}_{\bar{i}} d \mu \\
& +2 \int_{M}\left\{-v R|\nabla S|^{2}+v^{2} R^{2}|S|^{2}\right\} d \mu \\
= & 2 v^{2} \int_{M} R^{2}|S|^{2} d \mu-v \int_{M} R|\nabla S|^{2} d \mu \\
& +(2 v-1) \int_{M}|\nabla S|^{2} d \mu+(2 v-1) \int_{M} \dot{\varphi}_{i}\left\{S_{, k \bar{k}} \bar{S}_{\bar{i}}+S_{k} \bar{S}_{, \bar{i} \bar{k}}\right\} d \mu .
\end{aligned}
$$

Note that we used the property $S_{, l \bar{k}}=-v S R_{l \bar{k}}$. It follows that

$$
\begin{aligned}
\int_{M}|\nabla \nabla S|^{2} d \mu \leq & 2 v^{2} \int_{M} R^{2}|S|^{2} d \mu-v \int_{M} R|\nabla S|^{2} d \mu+(2 v-1) \int_{M}|\nabla S|^{2} d \mu \\
& -(2 v-1) v \int_{M} S R \dot{\varphi}_{i} \bar{S}_{\bar{i}} d \mu+(2 v-1) \int_{M} \bar{S}_{, \bar{i} \bar{k}} \dot{\varphi}_{i} S_{k} d \mu \\
\leq & C\left\{1+\int_{M}|\nabla S| d \mu+\int_{M}|\nabla \nabla S||\nabla S| d \mu\right\}
\end{aligned}
$$
(as $R, \dot{\varphi},|\nabla \dot{\varphi}|,|S|, \int_{M}|S|^{2} d \mu$ and $\int_{M}|\nabla S|^{2} d \mu$ are all bounded) $\leq C\left\{1+V+\int_{M}|\nabla S|^{2} d \mu+\frac{1}{2 C} \int_{M}|\nabla \nabla S|^{2} d \mu+2 C \int_{M}|\nabla S|^{2} d \mu\right\}$ (by the Hölder inequality and $x y \leq x^{2}+y^{2}$ ) $=\frac{1}{2} \int_{M}|\nabla \nabla S|^{2} d \mu+C\{1+V+(2 C+1) \mathcal{B} v\}$.

With a different $C$, we have proved that $\int_{M}|\nabla \nabla S|^{2} d \mu<C$ uniformly. On the other hand, we know

$$
\int_{M}|\bar{\nabla} \nabla S|^{2} d \mu=\int_{M} \nu^{2}|S|^{2}|\mathrm{Ric}|^{2} d \mu<C \int_{M}|\mathrm{Ric}|^{2} d \mu<C .
$$

Therefore the Sobolev inequality tells us that

$$
\begin{aligned}
\left(\int_{M}|\nabla S|^{\frac{2 n}{n-1}} d \mu\right)^{\frac{n-1}{n}} & \leq C_{S}\left\{\int_{M}|\nabla S|^{2} d \mu+\int_{M}|\nabla| \nabla S||^{2} d \mu\right\} \\
& \leq C\left\{\int_{M}|\nabla S|^{2} d \mu+\int_{M}|\nabla \nabla S|^{2} d \mu+\int_{M}|\bar{\nabla} \nabla S|^{2} d \mu\right\} .
\end{aligned}
$$


This means $\|\nabla S\|_{L^{2 n /(n-1)}}$ is uniformly bounded along the Kähler Ricci flow, proving Claim 3.

Fix $\beta>1$. Multiplying by $-|\nabla S|^{2(\beta-1)}$ both sides of (16) and integrating yields

$$
\begin{aligned}
\frac{4(\beta-1)}{\beta^{2}} & \left.\left.\int_{M}|\nabla| \nabla S\right|^{\beta}\right|^{2} d \mu \\
= & -\int_{M}\left(v^{2}|\mathrm{Ric}|^{2}|S|^{2}+|\nabla \nabla S|^{2}\right)|\nabla S|^{2(\beta-1)} d \mu+\int_{M} v R|\nabla S|^{2 \beta} d \mu \\
& +\underbrace{\int_{M}(2 v-1) R_{i \bar{k}} S_{k} \bar{S}_{\bar{i}}|\nabla S|^{2(\beta-1)} d \mu}_{I}+\underbrace{v \int_{M}\left\{S R_{i} \bar{S}_{\bar{i}}+\bar{S} R_{\bar{i}} S_{i}\right\}|\nabla S|^{2(\beta-1)} d \mu}_{I I} .
\end{aligned}
$$

Plugging $R_{i \bar{k}}=g_{i \bar{k}}-\dot{\varphi}_{i \bar{k}}$ into $I$ yields

$$
I=(2 v-1) \int_{M}|\nabla S|^{2 \beta} d \mu-(2 v-1) \int_{M} \dot{\varphi}_{i \bar{k}} S_{k} \bar{S}_{\bar{i}}|\nabla S|^{2(\beta-1)} d \mu .
$$

Since $S_{, l \bar{k}}=-v S R_{l \bar{k}}$, by the uniform bounds of $\dot{\varphi}, R$ and $|S|$, we have

$$
\begin{aligned}
\frac{I}{2 \nu-1}= & \int_{M}|\nabla S|^{2 \beta} d \mu-\int_{M} \dot{\varphi}_{i}\left(\nu R S \bar{S}_{\bar{i}}-S_{k} \bar{S}_{, \bar{i} \bar{k}}\right)|\nabla S|^{2(\beta-1)} d \mu \\
& +(\beta-1) \int_{M} \dot{\varphi}_{i} S_{k} \bar{S}_{\bar{i}}|\nabla S|^{2(\beta-2)}\left(-v S R_{l \bar{k}} \bar{S}_{\bar{l}}+S_{l} \bar{S}_{\overline{,} \bar{k}}\right) d \mu \\
\leq & \int_{M}|\nabla S|^{2 \beta} d \mu+C v \int_{M}|\nabla S|^{2 \beta-1} d \mu+C \int_{M}|\nabla \nabla S||\nabla S|^{2 \beta-1} d \mu \\
& +C(\beta-1) v \int_{M}|\operatorname{Ric}||S||\nabla S|^{2 \beta-1} d \mu+C(\beta-1) \int_{M}|\nabla \nabla S||\nabla S|^{2 \beta-1} .
\end{aligned}
$$

Therefore, for some constant $C$ (which may depend on $v$ ), we have

$$
I \leq C \int_{M}\left(|\nabla S|^{2 \beta-1}+|\nabla S|^{2 \beta}\right) d \mu+\beta C \int_{M}(\nu|\operatorname{Ric}||S|+|\nabla \nabla S|)|\nabla S|^{2 \beta-1} d \mu .
$$

Direct calculation shows

$$
\begin{aligned}
I I= & v \int_{M}\left\{-R|\nabla S|^{2 \beta}+v|S|^{2} R^{2}|\nabla S|^{2(\beta-1)}\right\} d \mu \\
& -v \int_{M} R S \bar{S}_{\bar{i}}(\beta-1)|\nabla S|^{2(\beta-2)}\left(-v \bar{S} R_{i \bar{l}} S_{l}+\bar{S}_{\bar{l}} S_{, l i}\right) d \mu \\
& +v \int_{M}\left\{-R|\nabla S|^{2 \beta}+v|S|^{2} R^{2}|\nabla S|^{2(\beta-1)}\right\} d \mu \\
& -v \int_{M} R \bar{S} S_{i}(\beta-1)|\nabla S|^{2(\beta-2)}\left(-v S R_{l \bar{l}} \bar{S}_{\bar{l}}+S_{l} \bar{S}_{, \overline{l i}}\right) d \mu
\end{aligned}
$$




$$
\begin{aligned}
= & -2 v \int_{M} R|\nabla S|^{2 \beta} d \mu+2 v^{2} \int_{M}|S|^{2} R^{2}|\nabla S|^{2(\beta-1)} d \mu \\
& +2(\beta-1) v^{2} \int_{M} R|S|^{2} R_{i \bar{l}} S_{l} \bar{S}_{\bar{i}}|\nabla S|^{2(\beta-2)} d \mu \\
& -(\beta-1) v \int_{M}\left\{S_{, l i} \bar{S}_{\bar{i}} \bar{S}_{\bar{l}} S+\bar{S}_{, \overline{l i}} S_{l} S_{i} \bar{S}\right\} R|\nabla S|^{2(\beta-2)} d \mu \\
\leq & C \int_{M}\left\{|\nabla S|^{2 \beta}+|\nabla S|^{2(\beta-1)}\right\} d \mu+\beta C \int_{M}(\nu|\operatorname{Ric}||S|+|\nabla \nabla S|)|\nabla S|^{2(\beta-1)} d \mu .
\end{aligned}
$$

Combining this estimate with the estimate of $I$ we have

$$
\begin{aligned}
&\left.\left.\frac{4(\beta-1)}{\beta^{2}} \int_{M}|\nabla| \nabla S\right|^{\beta}\right|^{2} d \mu \leq-\int_{M}\left(v^{2}|\operatorname{Ric}|^{2}|S|^{2}+|\nabla \nabla S|^{2}\right)|\nabla S|^{2(\beta-1)} d \mu \\
&+C \int_{M}\left\{|\nabla S|^{2 \beta}+|\nabla S|^{2(\beta-1)}\right\} d \mu \\
&+\beta C \int_{M}(\nu|\operatorname{Ric}||S|+|\nabla \nabla S|)\left\{|\nabla S|^{2(\beta-1)}+|\nabla S|^{2 \beta-1}\right\} d \mu .
\end{aligned}
$$

Since $\beta C \nu|\operatorname{Ric}||S||\nabla S|^{2(\beta-1)}=\left(\nu|\operatorname{Ric}||S||\nabla S|^{\beta-1}\right) \cdot\left(\beta C|\nabla S|^{\beta-1}\right)$, we see

$$
\begin{aligned}
\int_{M} \beta C \nu|\operatorname{Ric}||S||\nabla S|^{2(\beta-1)} d \mu \leq & \int_{M} \frac{1}{2} v^{2}|\operatorname{Ric}|^{2}|S|^{2}|\nabla S|^{2(\beta-1)} d \mu \\
& +\int_{M} \frac{1}{2}(\beta C)^{2}|\nabla S|^{2(\beta-1)} d \mu .
\end{aligned}
$$

Similar deduction yields

$$
\begin{aligned}
& \beta C \int_{M}(v|\operatorname{Ric}||S|+|\nabla \nabla S|)\left\{|\nabla S|^{2(\beta-1)}+|\nabla S|^{2 \beta-1}\right\} d \mu \\
& \quad \leq \int_{M}\left(v^{2}|\operatorname{Ric}|^{2}|S|^{2}+|\nabla \nabla S|^{2}\right)|\nabla S|^{2(\beta-1)} d \mu+\beta^{2} C^{2} \int_{M}\left\{|\nabla S|^{2(\beta-1)}+|\nabla S|^{2 \beta}\right\} d \mu .
\end{aligned}
$$

By adjusting the constant $C$, it follows from (18) that

$$
\left.\left.\frac{4(\beta-1)}{\beta^{2}} \int_{M}|\nabla| \nabla S\right|^{\beta}\right|^{2} d \mu \leq \beta^{2} C^{2} \int_{M}\left\{|\nabla S|^{2(\beta-1)}+|\nabla S|^{2 \beta}\right\} d \mu .
$$

If $\beta \geq n /(n-1)$, we have

$$
\left.\left.\int_{M}|\nabla| \nabla S\right|^{\beta}\right|^{2} d \mu \leq(C \beta)^{3} \int_{M}\left\{|\nabla S|^{2(\beta-1)}+|\nabla S|^{2 \beta}\right\} d \mu .
$$

The Sobolev inequality tells us that

$$
\begin{aligned}
\left(\int_{M}|\nabla S|^{\beta \cdot \frac{2 n}{n-1}}\right)^{\frac{n-1}{n}} & \leq C_{S}\left\{\int_{M}|\nabla S|^{2 \beta} d \mu+\left.\left.\int_{M}|\nabla| \nabla S\right|^{\beta}\right|^{2} d \mu\right\} \\
& \leq(2 C \beta)^{3} \int_{M}\left\{|\nabla S|^{2(\beta-1)}+|\nabla S|^{2 \beta}\right\} d \mu .
\end{aligned}
$$


From this inequality and the fact $\|\nabla S\|_{L^{2 n /(n-1)}}$ is uniformly bounded, the standard Moser iteration technique yields $\|\nabla S\|_{L^{\infty}}<A_{1}$ for some uniform constant $A_{1}$.

Corollary 3.2. Suppose $\left\{\left(M^{n}, g(t)\right), 0 \leq t<\infty\right\}$ is a Kähler Ricci flow solution. Then there is a constant $A_{2}$ depending only on this flow and $v$ such that $\left|\nabla F_{v}\right|<A_{2}$.

\subsection{Convergence of holomorphic sections of $K_{M}^{-v}$}

In this subsection we use the $L^{2}$-estimate for the $\bar{\partial}$-operator to study the convergence of pluri-anticanonical bundles. This section is very similar to Section 5 of Tian's paper [Tian90]. For the readers' and our convenience, we write down the arguments in detail.

First let us recall an important $\bar{\partial}$-lemma without proof.

Proposition 3.1 (cf. [Tian90, Proposition 5.1]). Suppose $\left(M^{n}, g, J\right)$ is a complete Kähler manifold, $\omega$ is a metric form compatible with $g$ and $J, L$ is a line bundle on $M$ with the Hermitian metric $h$, and $\psi$ is a smooth function on $M$. If

$$
\operatorname{Ric}(h)+\operatorname{Ric}(g)+\sqrt{-1} \partial \bar{\partial} \psi \geq c_{0} \omega
$$

for some uniform positive number $c_{0}$ at every point. Then for every smooth L-valued $(0,1)$-form $v$ on $M$ with $\bar{\partial} v=0$ and $\int_{M}|v|^{2} d \mu_{g}$ finite, there exists a smooth L-valued function $u$ on $M$ such that $\bar{\partial} u=v$ and

$$
\int_{M}|u|^{2} e^{-\psi} d \mu_{g} \leq \frac{1}{c_{0}} \int_{M}|v|^{2} e^{-\psi} d \mu_{g}
$$

where $|\cdot|$ is the norm induced by $h$ and $g$.

In our application, we fix $M$ to be a Fano manifold, and $L=K_{M}^{-v}$ for some integer $v$.

This proposition ensures that the plurigenera are continuous functions in a proper moduli space of complex varieties under the Cheeger-Gromov topology.

Theorem 3.1. Suppose $\left(M_{i}, g_{i}, J_{i}\right)$ is a sequence of Fano manifolds with the following properties:

(a) There is an a priori constant $\mathcal{B}$ such that

$$
C_{S}\left(M_{i}, g_{i}\right)+\left\|R_{g_{i}}\right\|_{C^{0}\left(M_{i}\right)}+\left\|u_{i}\right\|_{C^{0}\left(M_{i}\right)}<\mathcal{B} .
$$

Here $C_{S}\left(M_{i}, g_{i}\right)$ is the Sobolev constant of $\left(M_{i}, g_{i}\right), R_{g_{i}}$ is the scalar curvature, and $-u_{i}$ is the normalized Ricci potential, i.e.

$$
\operatorname{Ric}_{g_{i}}-\omega_{g_{i}}=-\sqrt{-1} \partial \bar{\partial} u_{i}, \quad \frac{1}{V_{g_{i}}} \int_{M_{i}} e^{-u_{i}} d \mu_{g_{i}}=1 .
$$

(b) There is a constant $K$ such that $K^{-1} r^{2 n} \leq \operatorname{Vol}(B(x, r)) \leq K r^{2 n}$ for every geodesic ball $B(x, r) \subset M_{i}$ satisfying $r \leq 1$.

(c) $\left(M_{i}, g_{i}, J_{i}\right) \stackrel{C^{\infty}}{\longrightarrow}(\hat{M}, \hat{g}, \hat{J})$ where $(\hat{M}, \hat{g}, \hat{J})$ is a $Q$-Fano metric-normal variety. 
Then for every positive integer $v$ such that $K_{\hat{M}}^{-v}$ is a well defined line bundle, we have:

1. If $S_{i} \in H^{0}\left(K_{M_{i}}^{-v}\right)$ and $\int_{M_{i}}\left|S_{i}\right|^{2} d \mu_{g_{i}}=1$, then by taking a subsequence if necessary, there is $\hat{S} \in H^{0}\left(K_{\hat{M}}^{-v}\right)$ such that

$$
S_{i} \stackrel{C^{\infty}}{\longrightarrow} \hat{S}, \quad \int_{\hat{M}}|\hat{S}|^{2} d \mu_{\hat{g}}=1 .
$$

2. If $\hat{S} \in H^{0}\left(K_{\hat{M}}^{-v}\right)$ and $\int_{\hat{M}}|\hat{S}|^{2} d \mu_{\hat{g}}=1$, then there is a subsequence of holomorphic sections $S_{i} \in H^{0}\left(K_{M_{i}}^{-v}\right)$ with $\int_{M_{i}}\left|S_{i}\right|^{2} d \mu_{g_{i}}=1$ such that $S_{i} \stackrel{C^{\infty}}{\longrightarrow} \hat{S}$.

Proof. For simplicity, we assume $K_{\hat{M}}^{-1}$ is a well defined line bundle over $\hat{M}$.

Let $\mathcal{P}$ be the singular set of $\hat{M}$. Since $\hat{M}$ is a Q-Fano metric-normal variety, by definition of Minkowski dimension, there is a constant $\mathcal{V}$ such that $\operatorname{Vol}(B(\mathcal{P}, r)) \leq \mathcal{V} r^{4}$ whenever $r$ is small. Now we prove parts 1 and 2 .

Part 1. According to the proof of Lemma 3.1, we see there is an a priori bound $A_{0}$ such that $\left|S_{i}\right|<A_{0}$.

Fix a small number $\delta$ and define $U_{\delta}=\hat{M} \backslash B(\mathcal{P}, \delta)$. By the definition of CheegerGromov convergence, there exists a sequence of diffeomorphisms $\phi_{i}: U_{\delta} \rightarrow \phi_{i}\left(U_{\delta}\right) \subset$ $M_{i}$ with the following properties:

(1) $\phi_{i}^{*} g_{i} \stackrel{C^{\infty}}{\longrightarrow} \hat{g}$ uniformly on $U_{\delta}$;

(2) $\left(\phi_{i}^{-1}\right)_{*} \circ J_{i} \circ\left(\phi_{i}\right)_{*} \stackrel{C^{\infty}}{\longrightarrow} \hat{J}$ uniformly on $U_{\delta}$.

Clearly, $\phi_{i}^{*} S_{i}$ is a section of $\left.\left(T^{(1,0)} \hat{M} \oplus T^{(0,1)} \hat{M}\right)\right|_{U_{\delta}}$ where $T^{(1,0)} \hat{M}$ and $T^{(0,1)} \hat{M}$ are defined according to the complex structure $\hat{J}$. Note that $\left|\phi_{i}^{*} S_{i}\right|_{C^{0}\left(U_{\delta}\right)}<A_{0}$ and $\phi_{i}^{*} S_{i}$ is holomorphic under the complex structure $\left(\phi_{i}^{-1}\right)_{*} \circ J_{i} \circ\left(\phi_{i}\right)_{*}$. By Cauchy's integration formula, all covariant derivatives of $\phi_{i}^{*} S_{i}$ with respect to $\phi_{i}^{*} g_{i}$ are uniformly bounded in the domain $U_{2 \delta}$. Therefore, there exists a section $\left.\hat{S}_{2 \delta} \in\left(T^{(1,0)} \hat{M} \oplus T^{(0,1)} \hat{M}\right)\right|_{U_{2 \delta}}$ such that $\phi_{i}^{*} S_{i} \stackrel{C^{\infty}}{\longrightarrow} \hat{S}_{2 \delta}$ on $U_{2 \delta}$. This section $\hat{S}_{2 \delta}$ is automatically holomorphic with respect to $\hat{J}$ since $\left(\phi_{i}^{-1}\right)_{*} \circ J_{i} \circ\left(\phi_{i}\right)_{*} \stackrel{C^{\infty}}{\longrightarrow} \hat{J}$ on $U_{2 \delta} \subset U_{\delta}$.

As $\left(M_{i}, g_{i}, J_{i}\right) \stackrel{C^{\infty}}{\longrightarrow}(\hat{M}, \hat{g}, \hat{J})$, we have $\lim _{i \rightarrow \infty} V_{g_{i}}\left(M_{i} \backslash \phi_{i}\left(U_{2 \delta}\right)\right)<2 \mathcal{V}(2 \delta)^{4}=$ $32 \mathcal{V} \delta^{4}$. It follows that

$$
\begin{aligned}
1 \geq \int_{U_{2 \delta}}\left|\phi_{i}^{*} S_{i}\right|^{2} d \mu_{\phi_{i}^{*} g_{i}} & =\int_{\phi_{i}\left(U_{2 \delta}\right)}\left|S_{i}\right|^{2} d \mu_{g_{i}}=\int_{M_{i}}\left|S_{i}\right|^{2} d \mu_{g_{i}}-\int_{M_{i} \backslash \phi_{i}\left(U_{2 \delta}\right)}\left|S_{i}\right|^{2} d \mu_{g_{i}} \\
& >1-32 A_{0}^{2} \mathcal{V} \delta^{4} .
\end{aligned}
$$

Therefore, for each $\delta$, there is a limit holomorphic section $\hat{S}_{2 \delta} \in H^{0}\left(K_{U_{2 \delta}}^{-1}\right)$ satisfying

$$
\left|\hat{S}_{2 \delta}\right|_{C^{0}\left(U_{2 \delta}\right)} \leq A_{0}, \quad 1 \geq \int_{U_{2 \delta}}\left|\hat{S}_{2 \delta}\right|^{2} d \mu_{\hat{g}} \geq 1-32 A_{0}^{2} \mathcal{V} \delta^{4} .
$$


Letting $\delta=\delta_{k}=2^{-k} \rightarrow 0$ and then taking a diagonal sequence, we obtain a subsequence of sections $\phi_{i_{k}}^{*} S_{i_{k}}$ satisfying

$$
\left.\left.\phi_{i_{k}}^{*} S_{i_{k}}\right|_{K} \stackrel{C^{\infty}}{\longrightarrow} \hat{S}\right|_{K}, \quad \forall \text { compact set } K \subset \hat{M} \backslash \mathcal{P}
$$

This exactly means that $\phi_{i_{k}}^{*} S_{i_{k}} \stackrel{C^{\infty}}{\longrightarrow} \hat{S}$ on $\hat{M} \backslash \mathcal{P}$. Moreover, we have $|\hat{S}|_{C^{0}(\hat{M} \backslash \mathcal{P})} \leq A_{0}$. Since $\hat{M}$ is a Q-Fano metric-normal variety, $\hat{S}$ can be naturally extended to a holomorphic section of $H^{0}\left(K_{\hat{M}}^{-1}\right)$. Clearly, we have

$$
\int_{\hat{M}}|\hat{S}|^{2} d \mu=\int_{\hat{M} \backslash \mathcal{P}}|\hat{S}|^{2} d \mu=1 .
$$

Part 2. Fix two small positive numbers $r, \delta$ satisfying $r \gg 2 \delta$. Define $\eta_{\delta}$ to be a cutoff function taking value 1 on $U_{2 \delta}$, and 0 on $B(\mathcal{P}, \delta)$, with $\left|\nabla \eta_{\delta}\right|_{\hat{g}}<2 / \delta$. Naturally, $\left(\phi_{i}\right)_{*}\left(\eta_{\delta} \hat{S}\right)$ can be viewed as a smooth section of the bundle $\Lambda^{n}\left(T^{(1,0)} M_{i} \oplus T^{(0,1)} M_{i}\right)$ by extension. Let $\pi_{i}$ be the projection from $\Lambda^{n}\left(T^{(1,0)} M_{i} \oplus T^{(0,1)} M_{i}\right)$ to $\Lambda^{n} T^{(1,0)} M_{i}$ and denote $V_{\delta, i}=\pi_{i}\left(\left(\phi_{i}\right)_{*}\left(\eta_{\delta} \hat{S}\right)\right)$. The smooth convergence of complex structures implies that $V_{\delta, i}$ is an almost holomorphic section of $\Lambda^{n} T^{(1,0)} M_{i}$. We have

$$
\lim _{i \rightarrow \infty} \sup _{\phi_{i}\left(U_{2 \delta}\right)}\left|\bar{\partial} V_{\delta, i}\right|=\lim _{i \rightarrow \infty} \sup _{\phi_{i}\left(U_{2 \delta}\right)}\left|\bar{\partial}\left(\pi_{i}\left(\left(\phi_{i}\right)_{*}\left(\eta_{\delta} \hat{S}\right)\right)\right)\right|=0
$$

where $\bar{\partial}$ is calculated under the complex structure $J_{i}$.

Notice that $V(B(\mathcal{P}, \delta)) \leq \mathcal{V} \delta^{4}$ for $\delta$ small. Defining $\mathcal{A}=|\hat{S}|_{C^{0}(\hat{M})}$, we have

$$
\begin{aligned}
1 & \geq \lim _{i \rightarrow \infty} \int_{M_{i}}\left|V_{\delta, i}\right|^{2} d \mu_{g_{i}}=\lim _{i \rightarrow \infty} \int_{M_{i}}\left|\pi_{i}\left(\left(\phi_{i}\right)_{*}\left(\eta_{\delta} \hat{S}\right)\right)\right|^{2} d \mu_{g_{i}} \geq 1-2 \mathcal{A}^{2} \mathcal{V}(2 \delta)^{4} \\
& =1-32 \mathcal{A}^{2} \mathcal{V} \delta^{4} .
\end{aligned}
$$

Recall that $V_{\delta, i}$ vanishes on $B(\mathcal{P}, \delta)$, so we have

$$
\int_{M_{i}}\left|\bar{\partial} V_{\delta, i}\right|^{2} d \mu_{g_{i}}=\int_{\phi_{i}\left(U_{2 \delta}\right)}\left|\bar{\partial} V_{\delta, i}\right|^{2} d \mu_{g_{i}}+\int_{\phi_{i}\left(U_{\delta} \backslash U_{2 \delta}\right)}\left|\bar{\partial} V_{\delta, i}\right|^{2} d \mu_{g_{i}} .
$$

By (20) and the inequalities $\left|\nabla \eta_{\delta}\right|_{\hat{g}}<2 / \delta$ and $\operatorname{Vol}\left(\phi_{i}\left(U_{\delta} \backslash U_{2 \delta}\right)\right) \leq 2 \mathcal{V}(2 \delta)^{4}$, we obtain

$$
\int_{M_{i}}\left|\bar{\partial} V_{\delta, i}\right|^{2} d \mu_{g_{i}} \leq 1000 \mathcal{A}^{2} \mathcal{V} \delta^{2}
$$

for large $i$.

Let $h_{i}$ be the Hermitian metric on $K_{M_{i}}^{-1}$ induced by $g_{i}$. Clearly, we have

$$
\operatorname{Ric}\left(h_{i}\right)+\operatorname{Ric}\left(g_{i}\right)+\sqrt{-1} \partial \bar{\partial}\left(-2 u_{i}\right)=2\left(\operatorname{Ric}\left(g_{i}\right)-\sqrt{-1} \partial \bar{\partial} u_{i}\right)=2 \omega_{g_{i}} .
$$


So we can to apply Proposition 3.1 to obtain a smooth section $W_{\delta, i}$ of $K_{M_{i}}^{-1}$ such that

$$
\left\{\begin{aligned}
\bar{\partial} W_{\delta, i}=\bar{\partial} V_{\delta, i}, & \\
\int_{M_{i}}\left|W_{\delta, i}\right|^{2} e^{2 u_{i}} d \mu_{g_{i}} & \leq \frac{1}{2} \int_{M_{i}}\left|\bar{\partial} V_{\delta, i}\right|^{2} e^{2 u_{i}} d \mu_{g_{i}} \leq \frac{e^{2 \mathcal{B}}}{2} \int_{M_{i}}\left|\bar{\partial} V_{\delta, i}\right|^{2} d \mu_{g_{i}} \\
& <500 \mathcal{A}^{2} \mathcal{V} e^{2 \mathcal{B}} \delta^{2} .
\end{aligned}\right.
$$

The triangle inequality implies

$$
\begin{aligned}
1+\sqrt{500 \mathcal{A}^{2} \mathcal{V} e^{2 \mathcal{B} \delta^{2}}} & >\left(\int_{M_{i}}\left|V_{\delta, i}-W_{\delta, i}\right|^{2} d \mu_{g_{i}}\right)^{1 / 2} \\
& >\sqrt{1-32 \mathcal{A}^{2} \mathcal{V} \delta^{4}}-\sqrt{500 \mathcal{A}^{2} \mathcal{V} e^{2 \mathcal{B} \delta^{2}}} .
\end{aligned}
$$

Therefore

$$
S_{\delta, i} \triangleq \frac{V_{\delta, i}-W_{\delta, i}}{\left(\int_{M_{i}}\left|V_{\delta, i}-W_{\delta, i}\right|^{2} d \mu_{g_{i}}\right)^{1 / 2}}
$$

is a well defined holomorphic section of $K_{M_{i}}^{-1}$.

Direct computation shows that $W_{\delta, i}$ satisfies the inequality

$$
\begin{aligned}
\Delta\left(\left|W_{\delta, i}\right|^{2}\right) & =\left|\nabla W_{\delta, i}\right|^{2}+\left|\bar{\nabla} W_{\delta, i}\right|^{2}-R\left|W_{\delta, i}\right|^{2}+2 \operatorname{Re}\left\langle W_{\delta, i}, \bar{\partial}^{*} \bar{\partial} W_{\delta, i}\right\rangle \\
& =\left|\nabla W_{\delta, i}\right|^{2}+\left|\bar{\nabla} W_{\delta, i}\right|^{2}-R\left|W_{\delta, i}\right|^{2}+2 \operatorname{Re}\left\langle W_{\delta, i}, \bar{\partial}^{*} \bar{\partial} V_{\delta, i}\right\rangle \\
& \geq\left|\nabla W_{\delta, i}\right|^{2}+\left|\bar{\nabla} W_{\delta, i}\right|^{2}-(R+1)\left|W_{\delta, i}\right|^{2}-\left|\bar{\partial}^{*} \bar{\partial} V_{\delta, i}\right|^{2} \\
& \geq\left|\nabla W_{\delta, i}\right|^{2}+\left|\bar{\nabla} W_{\delta, i}\right|^{2}-2 \mathcal{B}\left\{\left|W_{\delta, i}\right|^{2}+\frac{1}{2 \mathcal{B}}\left|\bar{\partial}^{*} \bar{\partial} V_{\delta, i}\right|^{2}\right\} .
\end{aligned}
$$

All geometric quantities are computed under the metric $g_{i}$ and complex structure $J_{i}$. Letting $f=\left|W_{\delta, i}\right|^{2}+\frac{1}{2 \mathcal{B}} \sup _{\phi_{i}\left(U_{\left.\frac{r}{2}\right)}\right.}\left|\bar{\partial}^{*} \bar{\partial} V_{\delta, i}\right|^{2}$, we have $\Delta f \geq-2 \mathcal{B} f$ on $\phi_{i}\left(U_{r / 2}\right)$. Applying local Moser iteration on $\phi_{i}\left(U_{r / 2}\right)$, we obtain

$$
\begin{aligned}
\|f\|_{C^{0}\left(\phi_{i}\left(U_{r}\right)\right)} & \leq C^{\prime}(r, \mathcal{B}, \mathcal{A})\|f\|_{L^{n /(n-1)}\left(\phi_{i}\left(U_{r / 2}\right)\right)} \\
& =C^{\prime}(r, \mathcal{B}, \mathcal{A})\left\{\left\|\left|W_{\delta, i}\right|^{2}\right\|_{L^{n /(n-1)}\left(\phi_{i}\left(U_{r / 2}\right)\right)}+\frac{1}{2 \mathcal{B}} \sup _{\phi_{i}\left(U_{r / 2}\right)}\left|\bar{\partial}^{*} \bar{\partial} V_{\delta, i}\right|^{2}\right\} .
\end{aligned}
$$

Since $\sup _{\phi_{i}\left(U_{r / 2}\right)}\left|\bar{\partial} * \bar{\partial} V_{\delta, i}\right|^{2}$ tends to 0 uniformly, we have

$$
\left\|\left|W_{\delta, i}\right|^{2}\right\|_{C^{0}\left(\phi_{i}\left(U_{r}\right)\right)} \leq C^{\prime \prime}(r, \mathcal{B}, \mathcal{A})\left\|\left|W_{\delta, i}\right|^{2}\right\|_{L^{n /(n-1)}\left(\phi_{i}\left(U_{r / 2}\right)\right)} .
$$

On the other hand, inequality (23) can be written as

$$
\left|\nabla W_{\delta, i}\right|^{2}+\left|\bar{\nabla} W_{\delta, i}\right|^{2} \leq \Delta\left(\left|W_{\delta, i}\right|^{2}\right)+2 \mathcal{B}\left|W_{\delta, i}\right|^{2}+\left|\bar{\partial} * \bar{\partial} V_{\delta, i}\right|^{2}
$$


Combining this with the Sobolev inequality, we can apply a cutoff function on $\phi_{i}\left(U_{r / 4} \backslash U_{r / 2}\right)$ to obtain

$$
\left\|\left|W_{\delta, i}\right|^{2}\right\|_{L^{n /(n-1)}\left(\phi_{i}\left(U_{r / 2}\right)\right)} \leq C^{\prime \prime \prime}(r, \mathcal{B}, \hat{M})\left\{\left\|\left|W_{\delta, i}\right|^{2}\right\|_{L^{1}\left(\phi_{i}\left(U_{r / 4}\right)\right)}+\sup _{\phi_{i}\left(U_{r / 4}\right)}\left|\bar{\partial}^{*} \bar{\partial} V_{\delta, i}\right|^{2}\right\} .
$$

Together with (24), the fact that $\sup _{\phi_{i}\left(U_{r / 4}\right)}\left|\bar{\partial} * \bar{\partial} V_{\delta, i}\right|^{2} \rightarrow 0$ implies that

$$
\begin{aligned}
\left\|\left|W_{\delta, i}\right|^{2}\right\|_{C^{0}\left(\phi_{i}\left(U_{r}\right)\right)} & \leq C^{\prime \prime \prime \prime}(r, \mathcal{B}, \mathcal{A}, \hat{M})\left\|\left|W_{\delta, i}\right|^{2}\right\|_{L^{1}\left(\phi_{i}\left(U_{r / 4}\right)\right)} \\
& \leq C^{\prime \prime \prime \prime}(r, \mathcal{B}, \mathcal{A}, \hat{M})\left\|\left|W_{\delta, i}\right|^{2}\right\|_{L^{1}\left(M_{i}\right)} \leq C(r, \mathcal{B}, \mathcal{A}, \mathcal{V}, \hat{M}) \delta^{2} .
\end{aligned}
$$

The last inequality follows from (21) and the fact that $\left|u_{i}\right|<\mathcal{B}$.

Fixing $r, \delta$ and letting $i \rightarrow \infty$, we have

$$
\lim _{i \rightarrow \infty} \phi_{i}^{*}\left(S_{\delta, i}\right)=\frac{\hat{S}+\hat{W}_{r}}{\lim _{i \rightarrow \infty}\left(\int_{M_{i}}\left|V_{\delta, i}-W_{\delta, i}\right|^{2} d \mu_{g_{i}}\right)^{1 / 2}}
$$

on the domain $U_{r}$, where $\hat{W}_{r}$ is a holomorphic section of $H^{0}\left(K_{U_{r}}^{-1}\right)$ with $\left\|\hat{W}_{r}\right\|_{C^{0}\left(U_{r}\right)}$ $\leq C \delta$. It follows from (22) and (25) that $\lim _{\delta \rightarrow 0} \lim _{i \rightarrow \infty} \phi_{i}^{*}\left(S_{\delta, i}\right)=\hat{S}$ on d $U_{r}$. Letting $\delta_{k}=2^{-k}$ and taking a diagonal sequence, we obtain $\lim _{k \rightarrow \infty} \phi_{i_{k}}^{*}\left(S_{2^{-k}, i_{k}}\right)=\hat{S}$ on $U_{r}$. Then letting $r=2^{-l}$ and taking a diagonal sequence once again, we obtain a sequence of holomorphic sections $S_{l} \triangleq S_{2-k_{l}, i_{k_{l}}}$ such that

$$
\lim _{l \rightarrow \infty} \phi_{l}^{*}\left(S_{l}\right)=\hat{S} \quad \text { on } \hat{M} \backslash \mathcal{P} .
$$

Since every $S_{l}$ is a holomorphic section (with respect to $\left.\left(\phi_{l}^{-1}\right)_{*} \circ J_{l} \circ\left(\phi_{l}\right)_{*}\right)$, Cauchy's integration formula implies that this convergence is actually in the $C^{\infty}$ topology.

\subsection{Criteria for tameness}

In this section, we show when the Kähler Ricci flow is tamed.

Theorem 3.2. Suppose $\left\{\left(M^{n}, g(t)\right), 0 \leq t<\infty\right\}$ is a Kähler Ricci flow satisfying the following conditions:

- Volume ratio bounded from above, i.e., there exists a constant $K$ such that

$$
\operatorname{Vol}_{g(t)}\left(B_{g(t)}(x, r)\right) \leq K r^{2 n}
$$

for every geodesic ball $B_{g(t)}(x, r)$ satisfying $r \leq 1$.

- Weak compactness, i.e., for every sequence $t_{i} \rightarrow \infty$, after passing to subsequence, we have

$$
\left(M, g\left(t_{i}\right)\right) \stackrel{C^{\infty}}{\longrightarrow}(\hat{M}, \hat{g}),
$$

where $(\hat{M}, \hat{g})$ is a $Q$-Fano metric-normal variety.

Then this flow is tamed. 
Proof. If this result is false, then for every $p_{i}=i$ !, $F_{p_{i}}$ is an unbounded function on $M \times[0, \infty)$. By Corollary $3.1, F_{p_{i}}$ has no lower bound. Therefore, there exists a point $\left(x_{i}, t_{i}\right)$ such that

$$
F_{p_{i}}\left(x_{i}, t_{i}\right)<-p_{i} .
$$

By weak compactness, we can assume that

$$
\left(M, g\left(t_{i}\right)\right) \stackrel{C^{\infty}}{\longrightarrow}(\hat{M}, \hat{g}) .
$$

Moreover, as $\hat{M}$ is a Q-Fano variety, we can assume $e^{\nu F_{\nu}(y)}=\sum_{\alpha=0}^{N_{v}}\left|S_{\nu, \alpha}(y)\right|_{\hat{\omega}^{\nu}}^{2}>c_{0}$ on $\hat{M}$. Applying Theorem 3.1 and Corollary 3.2, we have

$$
\lim _{i \rightarrow \infty} e^{\nu F_{v}\left(x_{i}, t_{i}\right)}>\frac{1}{2} c_{0}
$$

It follows that there are holomorphic sections $S_{v}^{\left(t_{i}\right)} \in H^{0}\left(K_{M}^{-v}\right)$ satisfying

$$
\int_{M}\left|S_{v}^{\left(t_{i}\right)}\right|_{h_{t_{i}}^{v}}^{2} \omega_{t_{i}}^{n}=1, \quad\left|S_{v}^{\left(t_{i}\right)}\right|_{h_{t_{i}}^{v}}^{2}\left(x_{i}\right)=e^{v F_{v}\left(x_{i}, t_{i}\right)}>\frac{1}{2} c_{0} .
$$

According to Lemma 3.1, there is a constant $C$ depending only on this flow such that

$$
\left|S_{v}^{\left(t_{i}\right)}\right|_{h_{i}^{v}}<C v^{n / 2}
$$

So we have

$$
A \triangleq \int_{M}\left|\left(S_{v}^{\left(t_{i}\right)}\right)^{k}\right|_{h_{t_{i}}^{k v}}^{2} \omega_{t_{i}}^{n}<V C^{2 k} v^{n k}
$$

Therefore, $A^{-1 / 2}\left(S_{v}^{\left(t_{i}\right)}\right)^{k}$ are unit sections of $H^{0}\left(K_{M}^{-k v}\right)$. It follows that

$$
\begin{aligned}
e^{k v F_{k v}\left(x_{i}, t_{i}\right)} & \geq\left|A^{-1 / 2}\left(S_{v}^{\left(t_{i}\right)}\right)^{k}\right|_{h_{t_{i}}^{k \nu}}^{2}\left(x_{i}\right) \geq V^{-1} C^{-2 k} v^{-n k}\left|\left(S_{\nu}^{\left(t_{i}\right)}\right)^{k}\right|_{h_{t_{i}}^{k v}}^{2}\left(x_{i}\right) \\
& \geq V^{-1} C^{-2 k} v^{-n k}\left(c_{0} / 2\right)^{k}
\end{aligned}
$$

This implies that

$$
k v \cdot F_{k v}\left(x_{i}, t_{i}\right) \geq-2 k \log C-n k \log v+k \log \left(c_{0} / 2\right)-\log V
$$

for large $i$ (depending on $v$ ) and every $k$. Let $k=p_{i} / v=i ! / \nu$. By (26), we have

$-k^{2} v^{2}=-p_{i}^{2}>p_{i} F_{p_{i}}\left(x_{i}, t_{i}\right)=k v \cdot F_{k v}\left(x_{i}, t_{i}\right) \geq-2 k \log C-n k \log v+k \log \left(c_{0} / 2\right)$.

However, this is impossible for large $k$.

In Theorem 4.4 of [CW3], we have proved the weak compactness of the Kähler Ricci flow on Fano surfaces, i.e., every sequence of evolving metrics of a Kähler Ricci flow solution on a Fano surface sub-converges to a KRS orbifold in the Cheeger-Gromov topology. Moreover, the volume ratio upper bound was proved as a lemma to prove the weak compactness. As an application of this property, we obtain 
Corollary 3.3. If $\left\{\left(M^{2}, g(t)\right), 0 \leq t<\infty\right\}$ is a Kähler Ricci flow on a Fano surface $M^{2}$, then it is tamed.

Proof. According to Theorem 4.4 of [CW3], every weak limit $(\hat{M}, \hat{g})$ is a KRS orbifold with finite singularities. It has positive first Chern class and it can be embedded into projective space by its pluri-anticanonical line bundle sections (cf. [Baily]). Clearly, finite singularities have Minkowski codimension 4 . Therefore, every $(\hat{M}, \hat{g})$ is a Q-Fano metricnormal variety, so Theorem 3.2 applies.

In [RZZ], Weidong Ruan, Yuguang Zhang and Zhenlei Zhang proved that the Riemannian curvature is uniformly bounded along the Kähler Ricci flow on $M^{n}(n \geq 3)$ if $\int_{M}|\mathrm{Rm}|^{n} d \mu$ is uniformly bounded. Under the latter condition, every sequential limit is a smooth Kähler Ricci soliton manifold, therefore Theorem 3.2 applies and we have

Corollary 3.4. Suppose $\left\{\left(M^{n}, g(t)\right), 0 \leq t<\infty\right\}$ is a Kähler Ricci flow along a Fano manifold $M^{n}$ and $n \geq 3$. If

$$
\sup _{0 \leq t<\infty} \int_{M}|\mathrm{Rm}|_{g(t)}^{n} d \mu_{g(t)}<\infty
$$

then the flow is tamed.

\section{The Kähler Ricci flow on Fano surfaces}

In this section, we give an application of the theorems we developed.

\subsection{Convergence of 2-dimensional Kähler Ricci flow}

Since the convergence of 2-dimensional Kähler Ricci flow was studied in [CW1] and [CW2] for all cases except $c_{1}^{2}(M)=1$ or 3 , we concentrate on these two remaining cases in this section.

Lemma 4.1. Suppose $M$ is a Fano surface.

- If $c_{1}^{2}(M)=1$, then $\alpha_{x}(S) \geq 5 /(6 v)$ for every $S \in H^{0}\left(K_{M}^{-v}\right)$ and $x \in M$.

- If $c_{1}^{2}(M)=3$, then $\alpha_{x}(S) \geq 2 /(3 v)$ for every $S \in H^{0}\left(K_{M}^{-v}\right)$ and $x \in M$. Moreover, if $\alpha_{x}\left(S_{1}\right)=\alpha_{x}\left(S_{2}\right)=2 /(3 v)$, then $S_{1}=\lambda S_{2}$ for some constant $\lambda$.

As a corollary, we have the following lemma.

Lemma 4.2. Suppose $M$ is a Fano surface.

- If $c_{1}^{2}(M)=1$, then $\alpha_{\nu, 1} \geq 5 / 6$.

- If $c_{1}^{2}(M)=3$, then $\alpha_{v, 1}=2 / 3$ and $\alpha_{v, 2}>2 / 3$. 
Because of Lemma 4.2 and Corollary 3.3, we can apply Theorem 2.2 or Theorem 2.3 respectively to obtain the following theorem.

Theorem 4.1. If $M$ is a Fano surface with $c_{1}^{2}(M)=1$ or 3 , then the Kähler Ricci flow on $M$ converges to a KE metric exponentially fast.

Combining this with the result in [CW1] and [CW2], we have proved the following result by the Ricci flow method.

Theorem 4.2. Every Fano surface $M$ has a KRS metric. This metric is a KE metric if and only if $\operatorname{Aut}(M)$ is reductive.

In particular, we have proved the Calabi conjecture on Fano surfaces by the flow method. This conjecture was first proved by Tian [Tian90] via the continuity method.

Remark 4.1. In [Chl], Cheltsov proved the following fact. Unless $M$ is a cubic surface with bad symmetry and with Eckardt point (a point lying on three exceptional lines), there exists a finite group $G$ such that $\alpha_{G}(M)>2 / 3$ for every $M$ satisfying $c_{1}^{2}(M) \leq 5$. Using this fact, we obtain the convergence of the Kähler Ricci flow on $M$ directly if $M \sim \mathbb{C P}^{2} \# 8 \overline{\mathbb{C P}}^{2}$. We thank Tian and Cheltsov for pointing this out to us. However, for consistency, we still give an independent proof for the convergence of the Kähler Ricci flow on $\mathbb{C P}^{2} \# 8 \overline{\mathbb{C P}}^{2}$ without applying this fact.

\subsection{Calculation of local $\alpha$-invariants}

In this subsection, we give an elementary proof of Lemma 4.1.

4.2.1. Local $\alpha$-invariants of holomorphic sections of $K_{M}^{-1}$

Proposition 4.1. Let $S \in H^{0}\left(\mathbb{C P}^{2}, 3 H\right), Z(S)$ be the divisor generated by $S$, and $x \in$ $Z(S)$. Then $\alpha_{x}(S)$ is totally determined by the singularity type of $x$. It is classified as in Table 1 below.

Proof. Direct computation.

Proposition 4.2. Suppose $M$ is a Fano surface with $M \sim \mathbb{C P}^{2} \# 6 \overline{C P}^{2}$, and $S \in H^{0}\left(K_{M}^{-1}\right)$. Then $\alpha_{x}(S) \geq 2 / 3$ for every $x \in Z(S)$. Moreover, if $S_{1}, S_{2} \in H^{0}\left(K_{M}^{-1}\right)$ and $\alpha_{x}\left(S_{1}\right)=$ $\alpha_{x}\left(S_{2}\right)=2 / 3$, then there exists a nonzero constant $\lambda$ such that $S_{1}=\lambda S_{2}$.

Proof. Let $M$ be the blowup of $\mathbb{C P}^{2}$ at points $p_{1}, \ldots, p_{6}$ in generic position. Let $\pi$ : $M \rightarrow \mathbb{C P}^{2}$ be the blowdown map. If $S \in H^{0}\left(K_{M}^{-1}\right)$, then $\pi_{*}(Z(S))$ must be a cubic curve $\gamma$ (maybe reducible) in $\mathbb{C P}^{2}$ and it must pass through every point $p_{i}$. It cannot contain any triple line. Indeed, assume it contains a triple line connecting $p_{1}$ and $p_{2}$. Then $Z(S)=3 H-a E_{1}-b E_{2}$ for some $a, b \in \mathbb{Z}^{+}$. On the other hand, we know $Z(S)=3 H-\sum_{i=1}^{6} E_{i}$, a contradiction.

Since no three $p_{i}$ 's are in the same line, a similar argument shows that there is no double line in $\pi_{*}(Z(S))$. 
Table 1. Local $\alpha$ invariants of holomorphic anticanonical sections on projective plane

\begin{tabular}{|c|l|c|}
\hline$\alpha_{x}(S)$ & Singularity type of $x$ & S's typical local equation \\
\hline \multirow{2}{*}{1} & smooth & $z$ \\
\cline { 2 - 3 } & transversal intersection of two smooth curves & $z w$ \\
\cline { 2 - 3 } & ordinary double point & $z^{2}-w^{2}(w+1)$ \\
\hline $5 / 6$ & cusp & $z^{3}-w^{2}$ \\
\hline $3 / 4$ & tangential intersection of a line and a conic curve & $z\left(z+w^{2}\right)$ \\
\hline $2 / 3$ & intersection of three different lines & $z w(z+w)$ \\
\hline $1 / 2$ & a point on a double line & $z^{2}$ \\
\hline $1 / 3$ & a point on a triple line & $z^{3}$ \\
\hline
\end{tabular}

So Table 1 implies $\alpha_{x}(S)=\alpha_{\pi(x)}\left(\pi_{*}(S)\right) \geq 2 / 3$ whenever $\pi(x) \in \mathbb{C P}^{2} \backslash\left\{p_{1}, \ldots, p_{6}\right\}$. Therefore we only need to consider singular points $x \in \pi^{-1}\left(\left\{p_{1}, \ldots, p_{k}\right\}\right)$. Without loss of generality, we assume $x \in \pi^{-1}\left(p_{1}\right)$ and $x$ is a singular point of $Z(S)$. We analyze this situation according to the singularity type of $\pi_{*}(x)$. Actually, $x$ is a singular point of $Z(S)$ only if $\pi_{*}(x)$ is a singular point of $\pi_{*}(Z(S))$. By Table 1 , we have the following classification.

1. $\pi_{*}(x)=p_{1}$ is an intersection point of three different lines. This case cannot happen: if such three lines exist, one of them must pass through three blowup points, impossible.

2. $\pi_{*}(x)$ is an intersection point of two different lines. In this case, $x$ must be a transversal intersection point of a curve and the exceptional divisor $E_{1}$. Therefore, $\alpha_{x}(S)=1$.

3. $\pi_{*}(x)$ is a cusp point. In this case, $x$ must be a tangential intersection point of a smooth curve and the exceptional divisor $E_{1}$. Moreover, the tangential order is just 1 . So $\alpha_{x}(S)=3 / 4$.

4. $\pi_{*}(x)$ is a tangential intersection point of a line and a conic curve. In this case, $x$ is a transversal intersection point of three curves $\gamma_{1}, \gamma_{2}, \gamma_{3}$ with $\left[\gamma_{1}\right]=E_{1},\left[\gamma_{2}\right] \sim$ $2 H-\sum_{l=1}^{6} E_{l}+E_{j},\left[\gamma_{3}\right] \sim H-E_{1}-E_{j}$ for some $j \in\{2, \ldots, 6\}$. Thus $x$ is an intersection point of three exceptional lines. Clearly, $\alpha_{x}(S)=2 / 3$.

Therefore, $\alpha_{x}(S) \geq 2 / 3$ for any $x \in \pi^{-1}\left\{p_{1}, \ldots, p_{6}\right\}$. Moreover, $\alpha_{x}(S)=2 / 3$ only if $x$ is a transversal intersection point of three exceptional lines.

It is well known that $M$ is a cubic surface, and there are in total 27 exceptional lines on $M$. Each point can lie on at most three exceptional lines. Therefore, if $\alpha_{x}\left(S_{1}\right)=$ $\alpha_{x}\left(S_{2}\right)=2 / 3$, then $Z\left(S_{1}\right)=Z\left(S_{2}\right)$ as the union of three exceptional lines passing through $x$. So there is a nonzero constant $\lambda$ such that $S_{1}=\lambda S_{2}$.

Proposition 4.3. Suppose $M$ is a Fano surface with $M \sim \mathbb{C P}^{2} \# 8 \overline{\mathbb{C P}}^{2}$, and $S \in H^{0}\left(K_{M}^{-1}\right)$. Then $\alpha_{x}(S) \geq 5 / 6$ for every $x \in Z(S)$.

Proof. In the notation of the proof of Proposition 4.2, we see $\pi_{*}(Z(S))$ is a cubic curve. Suppose $\pi_{*}(Z(S))$ is reducible; then $Z(S)=\gamma_{1}+\gamma_{2}$ with $\gamma_{1}$ a line and $\gamma_{2}$ a conic curve. 
So $Z(S)$ can pass through at most $2+5=7$ blowup points. On the other hand, it must pass through all of them, a contradiction. Therefore, $\pi_{*}(Z(S))$ is irreducible.

If $\pi(x) \in \mathbb{C P}^{2} \backslash\left\{p_{1}, \ldots, p_{8}\right\}$, we have $\alpha_{x}(S)=\alpha_{\pi(x)}\left(\pi_{*}(S)\right) \geq 5 / 6>2 / 3$ by Table 1. Suppose $\pi(x) \in\left\{p_{1}, \ldots, p_{8}\right\}$. These eight points are in generic position. No cubic curve passes through seven of them with one point doubled. Since $\pi_{*}(Z(S))$ is a cubic curve passing through all of these eight points, it must pass through every point smoothly. It follows that $x$ is a smooth point of $Z(S)$. So $\alpha_{x}(S)=1$.

\subsubsection{Local $\alpha$-invariants of holomorphic sections of $K_{M}^{-v}$}

Proposition 4.4. If $f, g$ are holomorphic functions (or holomorphic sections of a line bundle) defined in a neighborhood of $x$, then $\alpha_{x}(f g) \geq \frac{\alpha_{x}(f) \alpha_{x}(g)}{\alpha_{x}(f)+\alpha_{x}(g)}$, i.e.,

$$
\frac{1}{\alpha_{x}(f g)} \leq \frac{1}{\alpha_{x}(f)}+\frac{1}{\alpha_{x}(g)}
$$

Proof. Without loss of generality, we can assume $\alpha_{x}(f), \alpha_{x}(g)<\infty$. For simplicity of notation, let $a=\alpha_{x}(f), b=\alpha_{x}(g), c=\frac{a b}{a+b}$. We only need to prove $\alpha_{x}(f g) \geq c$.

Fix a small number $\epsilon>0$. Since $c / a+c / b=1$, the Hölder inequality implies

$$
\int_{U}(f g)^{-2 c(1-\epsilon)} d \mu=\left(\int_{U} f^{-2 a(1-\epsilon)} d \mu\right)^{c / a}\left(\int_{U} g^{-2 b(1-\epsilon)}\right)^{c / b}<\infty
$$

where $U$ is some neighborhood of $x$. Therefore, $\alpha_{x}(f g) \geq c(1-\epsilon)$. As $\epsilon$ can be arbitrarily small, we have $\alpha_{x}(f g) \geq c$.

As an application of Proposition A.1.1 of [Tian90], we state the following property without proof.

Proposition 4.5. Suppose $f$ is a holomorphic function vanishing at $x$ with order $k$. In a small neighborhood, we can express $f$ as

$$
f=a_{i j} z_{1}^{i} z_{2}^{j}+\cdots
$$

Without loss of generality, we can assume that there is a pair $(i, j)$ such that $i \geq j$, $i+j=k$ and $a_{i j} \neq 0$. Then $\alpha_{x}(f) \geq 1 / i$.

Lemma 4.3. Suppose $M$ is a cubic surface, and let $S \in H^{0}\left(K_{M}^{-m}\right), x \in M$. If $\alpha_{x}(S) \leq$ $2 /(3 m)$, then $\alpha_{x}(S)=2 /(3 m)$ and $S=\left(S^{\prime}\right)^{m}$ where $S^{\prime} \in H^{0}\left(K_{M}^{-1}\right)$ and $Z\left(S^{\prime}\right)$ is the union of three lines passing through $x$.

Proof. We will prove this statement by induction. Suppose we have already proved it for all $k \leq m-1$; now we show it is true for $k=m$.

Claim 1. If $S$ splits off an anticanonical holomorphic section $S^{\prime}$, then $Z\left(S^{\prime}\right)$ must be the union of three lines passing through $x$. Moreover, $S=\left(S^{\prime}\right)^{m}$. 
Suppose $S=S^{\prime} S_{m-1}$ where $S^{\prime} \in H^{0}\left(K_{M}^{-1}\right)$ and $S_{m-1} \in H^{0}\left(K_{M}^{-(m-1)}\right)$. Since $\alpha_{x}\left(S^{\prime}\right) \geq$ $2 / 3$ and $\alpha_{x}\left(S_{m-1}\right) \geq 2 /(3(m-1))$, by induction assumption, inequality (27) implies

$$
\frac{3 m}{2} \leq \frac{1}{\alpha_{x}(S)} \leq \frac{1}{\alpha_{x}\left(S^{\prime}\right)}+\frac{1}{\alpha_{x}\left(S_{m-1}\right)} \leq \frac{3}{2}+\frac{3(m-1)}{2}=\frac{3 m}{2} .
$$

This forces that

$$
\alpha_{x}(S)=\frac{2}{3 m}, \quad \alpha_{x}\left(S^{\prime}\right)=\frac{2}{3}, \quad \alpha_{x}\left(S_{m-1}\right)=\frac{2}{3(m-1)} .
$$

Therefore the induction hypothesis tells us that $Z\left(S^{\prime}\right)$ is the union of three lines passing through $x, S_{m-1}=\left(S^{\prime \prime}\right)^{m-1}$ and $Z\left(S^{\prime \prime}\right)$ is the union of three lines passing through $x$. As there are at most three lines passing through $x$ on a cubic surface, we see $Z\left(S^{\prime}\right)=Z\left(S^{\prime \prime}\right)$. By changing coefficients if necessary, we have $S_{m-1}=\left(S^{\prime}\right)^{m-1}$. It follows that $S=\left(S^{\prime}\right)^{m}$ and we have finished the proof.

Claim 2. There must be a line passing through $x$.

Otherwise, there is a pencil of anticanonical divisors passing through $x$. In this pencil, a generic divisor is irreducible and it vanishes at $x$ to order 2. Choose such a divisor and denote it by $Z\left(S^{\prime}\right)$. Locally, we can represent $S$ by a holomorphic function $f$. Since $\alpha_{x}(f)=\alpha_{x}(S) \leq 2 /(3 m)$, we see mult $_{x}(f) \geq\lceil 3 m /(2)\rceil$. If $m$ is odd, then $Z\left(S^{\prime}\right) \nsubseteq Z(S)$ implies

$$
3 m=K_{M}^{-m} \cdot K_{M}^{-1} \geq 2 \operatorname{mult}_{x}(f) \geq 3 m+1,
$$

impossible. Since $Z\left(S^{\prime}\right)$ is irreducible, we have $Z\left(S^{\prime}\right) \subset Z(S)$. Therefore, $S=S^{\prime} S_{m-1}$. According to Claim $1, Z\left(S^{\prime}\right)$ is the union of three lines and therefore $Z\left(S^{\prime}\right)$ is reducible. This contradicts the assumption on $Z\left(S^{\prime}\right)$.

So $m$ must be an even number and $\operatorname{mult}_{x}(f)=3 m / 2$ exactly. Now $f$ can be written as

$$
\sum_{i, j \geq 0} a_{i j} z_{1}^{i} z_{2}^{j}, \quad a_{i j}=0 \quad \text { whenever } i+j<3 m / 2 .
$$

Using the fact that $\alpha_{x}(f) \leq 2 /(3 m)$, Proposition 4.5 implies $a_{i j}=0$ whenever $i<3 m / 2$, and $a_{3 m / 2,0} \neq 0$. Therefore locally $f$ can be written as $a_{3 m / 2,0} z_{1}^{3 m / 2} \cdot h$ for some nonzero holomorphic function $h$. This means that $Z(S)$ contains a curve $\gamma$ with multiplicity $3 m / 2$. Project $Z(S)$ to $\mathbb{C P}^{2}$ by the map $\pi$. The image curve $\pi(\gamma)$ must be of degree one in $\mathbb{C P}^{2}$, since otherwise $\pi(Z(S))=\pi(\gamma)$ does not pass through other blowup points. Since $\pi(\gamma)$ is of degree one, it can only pass through two of the blowup points. Moreover, it vanishes at each of these two points to order $3 m / 2>m$, impossible.

Claim 3. The number of lines passing through $x$ is greater than 1.

Otherwise, there is exactly one line $L_{1}$ passing through $x$. So there is an irreducible degree 2 curve $D$ passing through $x$ such that $L_{1}+D=Z\left(S^{\prime}\right)$ for some $S^{\prime} \in H^{0}\left(K_{M}^{-1}\right)$. Locally, we can write $S$ as $l_{1} h$ where $l_{1}$ is the defining function for $L_{1}$. As $\alpha_{x}\left(l_{1}\right)=1$, 
the Hölder inequality implies that $\alpha_{x}(h) \leq 2 /(3 m-2)$. Consequently, $\operatorname{mult}_{x}(h) \geq$ $\lceil 3 m / 2\rceil-1$. If $2 L_{1} \nsubseteq Z(S)$, we have

$$
m+1=\left(K_{M}^{-m}-L_{1}\right) \cdot L_{1} \geq\{h=0\} \cdot L_{1} \geq\lceil 3 m / 2\rceil-1, \quad \Leftrightarrow m \leq 4 .
$$

If $m>4$, this inequality is wrong so we have $2 L_{1} \subset Z(S)$. Actually, using this argument and induction, we can show that $\lceil m / 4\rceil L_{1} \subset Z(S)$.

For simplicity of notation, let $p=\lceil m / 4\rceil$. Locally, $S$ can be written as $l_{1}^{p} h$. Clearly, $\alpha_{x}(h) \leq 2 /(3 m-2 p)$ and $\operatorname{mult}_{x}(h) \geq\lceil 3 m / 2\rceil-p$. Let $f_{q}$ and $h_{q-2}$ be the lowest degree terms of $f$ and $h$ respectively. Then we may assume that $h_{q-2}=z_{1}^{j_{1}} z_{2}^{j_{2}}+\cdots$ and any term $z_{1}^{i} z_{2}^{j}$ in $h_{q-2}$ satisfies $i \geq j_{1}$. Now we have two cases to consider.

Case 1: $L_{1}$ is tangent to $\left\{z_{1}=0\right\}$. If $(p+1) L_{1} \nsubseteq Z(S)$, then

$m+p=\left(K_{M}^{-m}-p L_{1}\right) \cdot L_{1} \geq\{h=0\} \cdot L_{1} \geq(\lceil 3 m / 2\rceil-p) \cdot 2, \quad \Leftrightarrow p \geq \frac{m+2\lceil m / 2\rceil}{3}$.

Here we use the fact that $j_{1} \geq\lceil 3 m / 2\rceil-p$ since $\alpha_{x}(h) \leq 2 /(3 m-2 p)$. This contradicts our definition $p=\lceil m / 4\rceil$. Therefore, $(p+1) L_{1} \subset Z(S)$.

Case 2. $L_{1}$ is not tangent to $\left\{z_{1}=0\right\}$. In this case, $f_{q}=\lambda z_{1}^{a_{1}} z_{2}^{a_{2}+p}+\cdots$ for some $\lambda \neq 0$. Moreover, every $z_{1}^{i} z_{2}^{j}$ in $f_{q}$ satisfies $i \geq j_{1}$. Therefore, the fact that $\alpha_{x}(S) \leq 2 /(3 m)$ and Proposition 4.5 imply $a_{1} \geq\lceil 3 m / 2\rceil$. It follows that $q \geq p+\lceil 3 m / 2\rceil$. Under these conditions, if $(p+1) L_{1} \nsubseteq \bar{Z}(S)$, we have

$$
m+p=\left(K_{M}^{-m}-p L_{1}\right) \cdot L_{1} \geq\lceil 3 m / 2\rceil, \quad \Leftrightarrow p \geq\lceil m / 2\rceil .
$$

If $m \geq 3$ this is impossible, so $(p+1) L_{1} \subset Z(S)$. If $m \leq 2$, as $\lceil m / 2\rceil=\lceil m / 4\rceil=1$, we already know $\lceil m / 2\rceil L_{1} \subset Z(S)$.

Therefore by repeatedly rewriting $S$ in local charts and considering Cases 1 and 2, we can prove $\lceil m / 2\rceil L_{1} \subset Z(S)$. Furthermore, we have proved the following property.

- Suppose $S$ can be written as $l_{1}^{n} h^{\prime}$ locally with $n=\lceil m / 2\rceil$. Then either $(n+1) L_{1} \subset$ $Z(S)$ or $L_{1}$ is not tangent to $\left\{z_{1}=0\right\}$.

From this property we can show $D \subset Z(S)$. In fact, if $(n+1) L_{1} \subset Z(S)$ and $D \nsubseteq Z(S)$, we have

$$
\begin{aligned}
2 m & =K_{M}^{-m} \cdot D \geq(n+1) L_{1} \cdot D+\operatorname{mult}_{x}\left(h^{\prime \prime}\right) \\
& \geq 2(n+1)+(\lceil 3 m / 2\rceil-(n+1)), \quad \Leftrightarrow m \geq 2 n+1,
\end{aligned}
$$

where $h^{\prime \prime}$ is a function such that locally $S$ is represented by $l_{1}^{n+1} h^{\prime \prime}$. This inequality is impossible as $n=\lceil m / 2\rceil$. If $L_{1}$ is not tangent to $\left\{z_{1}=0\right\}$, we know $\operatorname{mult}_{x}\left(h^{\prime}\right) \geq$ $n+\lceil 3 m / 2\rceil=m+2 n$. Therefore, $D \nsubseteq Z(S)$ implies that

$$
2 m=K_{M}^{-m} \cdot D \geq n L_{1} \cdot D+\operatorname{mult}_{x}\left(h^{\prime}\right) \geq m+4 n, \quad \Leftrightarrow m \geq 4\lceil m / 2\rceil,
$$

impossible. Therefore, no matter which case happens, we have $D \subset Z(S)$. So $D+L_{1} \subset$ $Z(S)$. It follows that $S$ splits off an $S^{\prime} \in H^{0}\left(K_{M}^{-1}\right)$ with $Z\left(S^{\prime}\right)=L_{1}+D$, contrary to Claim 1. 
Claim 4. The number of lines passing through $x$ is greater than 2.

Otherwise, there are only two lines $L_{1}$ and $L_{2}$ passing through $x$. There is a unique line $L_{3}$ not passing through $x$ such that $L_{1}+L_{2}+L_{3} \in K_{M}^{-1}$. We first prove the following property:

$$
k\left(L_{1}+L_{2}\right) \subset Z(S) \quad \text { for all } 0 \leq k \leq n=\lceil m / 2\rceil .
$$

By induction, we can assume $(k-1)\left(L_{1}+L_{2}\right) \in Z(S)$. Then $S$ can be represented by a holomorphic function $f=l_{1}^{k-1} l_{2}^{k-1} h$ locally. Noting that $\alpha_{x}\left(l_{1}^{k-1} l_{2}^{k-1}\right)=1 /(k-1)$, the Hölder inequality implies

$$
\alpha_{x}(h) \leq \frac{\frac{2}{3 m}}{1-\frac{2(k-1)}{3 m}}=\frac{2}{3 m-2(k-1)} .
$$

It follows that

$$
\operatorname{mult}_{x}(h) \geq\lceil 3 m / 2\rceil+1-k=m+n+1-k .
$$

If $k L_{1} \nsubseteq Z(S)$, we have

$$
\begin{aligned}
m & =\left(K_{M}^{-m}-(k-1)\left(L_{1}+L_{2}\right)\right) \cdot L_{1} \geq\{h=0\} \cdot\left\{l_{1}=0\right\} \\
& \geq \operatorname{mult}_{x}(h) \geq m+n+1-k, \quad \Leftrightarrow k \geq n+1 .
\end{aligned}
$$

This contradicts the assumption on $k$. Therefore, $k L_{1} \subset Z(S)$. Similarly, $k L_{2} \subset Z(S)$. So $k\left(L_{1}+L_{2}\right) \subset Z(S)$.

Now locally $S$ can be written as $l_{1}^{n} l_{2}^{n} h$. We have $\alpha_{x}(h) \leq 2 /(3 m-2 n)$ and $\operatorname{mult}_{x}(h)$ $\geq\lceil 3 m / 2-n\rceil=m$. Assume mult $x_{x}(h)=m$. In local coordinates, $h=\sum_{i, j \geq 0} a_{i j} z_{1}^{i} z_{2}^{j}$. Since $\alpha_{x}(h) \leq 2 /(3 m-2 n)$, Proposition 4.5 implies $a_{i j}=0$ whenever $i<\lceil 3 m / 2-n\rceil$ $=m$. Since mult $_{x}(h)=m$, we see that the lowest homogeneous term of $f$ is of the form $l_{1}^{n} l_{2}^{n} z_{1}^{m}$. The condition $\alpha_{x}(S) \leq 2 /(3 m)<1 / m$ implies that either $L_{1}$ or $L_{2}$, say $L_{1}$, is tangent to $\left\{z_{1}=0\right\}$ at $x$. If $(n+1) L_{1} \nsubseteq Z(S)$, we have

$$
\begin{aligned}
m+n & =\left(K_{M}^{-m}-n L_{1}\right) \cdot L_{1} \geq\left\{l_{2}^{n} h=0\right\} \cdot L_{1} \\
& \geq n+\left\{\sum_{i, j \geq 0} a_{i j} z_{1}^{i} z_{2}^{j}=0\right\} \cdot L_{1} \geq n+\inf \left\{2 i+j \mid a_{i j} \neq 0\right\} \geq n+2 m,
\end{aligned}
$$

impossible. It follows that $(n+1) L_{1}+n L_{2} \subset Z(S)$.

Now we consider $L_{3}$. If $L_{3} \nsubseteq Z(S)$, we have

$$
m=L_{3} \cdot K_{M}^{-m} \geq\left((n+1) L_{1}+n L_{2}\right)=2 n+1=2\lceil m / 2\rceil+1 .
$$

This absurd inequality implies $L_{3} \subset Z(S)$. Let $S^{\prime} \in K_{M}^{-1}$ be such that $Z\left(S^{\prime}\right)=L_{1}+$ $L_{2}+L_{3}$. We have $S=S^{\prime} S_{m-1}$. However, $Z\left(S^{\prime}\right)$ is not the union of three lines passing through $x$. This contradicts Claim 1 .

Thus, there exist three lines $L_{1}, L_{2}, L_{3} \subset Z(S)$ passing through $x$. Since $M$ is a cubic surface, there exists an $S^{\prime} \in H^{0}\left(K_{M}^{-1}\right)$ such that $Z\left(S^{\prime}\right)=L_{1}+L_{2}+L_{3}$. As we argued in Claim 4, $L_{1}, L_{2}, L_{3} \subset Z(S)$. Therefore $L_{1}+L_{2}+L_{3} \subset Z(S)$ and $S$ splits off an anticanonical holomorphic section $S^{\prime}$. By Claim 1, we have $S=\left(S^{\prime}\right)^{m}$.

Similarly, we can prove the following property by induction. 
Lemma 4.4. Suppose $M$ is a Fano surface with $M \sim \mathbb{C P}^{2} \# 8 \overline{\mathbb{C P}}^{2}$, and let $S \in H^{0}\left(K_{M}^{-m}\right)$ and $x \in M$. Then $\alpha_{x}(S) \geq 5 /(6 m)$ for every $x \in M$.

Proof. Suppose we have proved this statement for all $k \leq m-1$.

Suppose this statement is wrong for $k=m$. Then there is a holomorphic section $S \in$ $H^{0}\left(K_{M}^{-m}\right)$ and a point $x \in M$ such that $\alpha_{x}(S)<5 /(6 m)$. Let $f$ be a local holomorphic function representing $S$. Clearly, $\operatorname{mult}_{\chi}(f)>6 \mathrm{~m} / 5$. Choose $S^{\prime} \in H^{0}\left(K_{M}^{-1}\right)$ such that $x \in Z\left(S^{\prime}\right)$. Since $S^{\prime}$ is irreducible, if $Z\left(S^{\prime}\right) \nsubseteq Z(S)$, we have

$$
m=Z(S) \cdot Z\left(S^{\prime}\right)>6 m / 5,
$$

impossible. Therefore, $Z\left(S^{\prime}\right) \subset Z(S)$. It follows that $S=S^{\prime} S_{m-1}$ for some $S_{m-1} \in$ $H^{0}\left(K_{M}^{-1}\right)$. So Proposition 4.5 implies

$$
\alpha_{x}(S) \geq \frac{\frac{5}{6} \cdot \frac{5}{6(m-1)}}{\frac{5}{6}+\frac{5}{6(m-1)}}=\frac{5}{6 m},
$$

contrary to the assumption on $\alpha_{x}(S)$.

Lemma 4.1 is a combination of Lemmas 4.3 and 4.4.

Acknowledgments. This work benefits from [Tian90] conceptually. The second named author is very grateful to G. Tian for many insightful and inspiring conversations. He would also like to thank J. Cheeger, B. Chow, K. Grove, and J.-P. Bourguignon for their interest in this work. The first named author would like to thank S. K. Donaldson for lengthy discussions on this and related projects in Kähler geometry. Both authors are grateful to the referee for many helpful suggestions.

The research of X. X. Chen was partially supported by an NSF grant.

\section{References}

[Baily] Baily, W. L.: On the imbedding of V-manifolds in projective space. Amer. J. Math. 79, 403-430 (1957) Zbl 0173.22706 MR 0100104

[Ca] Calabi, E.: The space of Kähler metrics. In: Proceedings of the International Congress of Mathematicians (Amsterdam, 1954), Vol. II, Noordhoff, Groningen, 206-207 (1954)

[Cao85] Cao, H. D.: Deformation of Kähler metrics to Kähler-Einstein metrics on compact Kähler manifolds. Invent. Math. 81, 359-372 (1985) Zbl 0574.53042 MR 0799272

[Chl] Cheltsov, I.: Log canonical threshholds of Del Pezzo surfaces. Geom. Funct. Anal. 18, 1118-1144 (2008) Zbl 1161.14030 MR 2465686

[CH] Chen, X. X., Li, H. Z.: Stability of Kähler Ricci flow. J. Geom. Anal. 20, 306-334 (2010) Zbl 1185.53074 MR 2579512

[CST] Chen, X. X., Sun, S., Tian, G.: A note on Kähler-Ricci soliton. Int. Math. Res. Notices 2009, 3328-3336 Zbl 1177.53037 MR 2535001

[CT1] Chen, X. X., Tian, G.: Ricci flow on Kähler-Einstein surfaces. Invent Math. 147, 487544 (2002) Zbl 1047.53043 MR 1893004

[CT2] Chen, X. X., Tian, G.: Ricci flow on Kähler-Einstein manifolds, Duke Math. J. 131, 17-73 (2006) Zbl 1097.53045 MR 2219236

[CW1] Chen, X. X., Wang, B.: The Kähler Ricci flow on Fano surfaces (I). Math. Z. 270, $577-$ 587 (2012) Zbl 1237.53066 MR 2875850 
[CW2] Chen, X. X., Wang, B.: Remarks on Kähler Ricci flow. J. Geom. Anal. 20, 335-353 (2010) Zbl 1185.53075 MR 2579513

[CW3] Chen, X. X., Wang, B.: Space of Ricci flows (I). arXiv:0902.1545

[Di] Ding, W.: Remarks on the existence problem of positive Kähler-Einstein metrics. Math. Ann. 282, 463-471 (1982) Zbl 0661.53045 MR 0967024

[DK] Demailly, J.-P., Kollár, J.: Semi-continuity of complex singularity exponents and Kähler Einstein metrics on Fano orbifolds. Ann. Sci. École Norm. Sup. (4) 34, 525-556 (2001) Zbl 0994.32021 MR 1852009

[DT] Ding, W. Y., Tian, G.: Kähler-Einstein metrics and the generalized Futaki invariants. Invent. Math. 110, 315-335 (1992) Zbl 0779.53044 MR 1185586

[Fu] Futaki, A.: An obstruction to the existence of Einstein Kähler metrics. Invent. Math. 73, 437-443 (1983) Zbl 0506.53030 MR 0718940

[Hei] Heier, G.: Convergence of the Kähler Ricci flow and multiplier ideal sheaves on Del Pezzo surfaces. Michigan Math. J. 58, 423-440 (2009) Z Zbl 1187.53069 MR 2595547

[Kosta] Kosta, D.: Del Pezzo surfaces with Du Val singularities. arXiv: 0904.0943v1

[Ma] Matsushima, Y.: Sur la structure du groupe d'homéomorphismes analytiques d'une certaine variété Kaehlérienne. Nagoya Math. J. 11, 145-150 (1957) Zbl 0091.34803 MR 0094478

[Pe] Perelman, G.: The entropy formula for the Ricci flow and its geometric applications. arXiv:math.DG/0211159 Zbl 1130.53001

[PSS] Phong, D. H., Sesum, N., Sturm, J.: Mutiplier ideal sheaves and the Kähler Ricci flow. Comm. Anal. Geom. 15, 613-632 (2007) Zbl 1143.53064 MR 2379807

[PSSW1] Phong, D. H., Song, J., Sturm, J., Weinkove, B.: The Kähler Ricci flow with positive bisectional curvature. Invent. Math. 173, 651-665 (2008) Zbl 1145.53050 MR 2425138

[PSSW2] Phong, D. H., Song, J, Sturm, J., Weinkove, B.: The Kähler Ricci Flow and the $\bar{\partial}$ operator on vector fields. J. Differential Geom. 81, 631-647 (2009) Zbl 1162.32014 MR 2487603

[PS] Phong, D. H., Sturm, J.: Algebraic estimates, stability of local zeta functions, and uniform estimates for distribution functions. Ann. of Math. (2) 152, 277-329 (2000) Zbl 0995.11065 MR 1792297

[RZZ] Ruan, W. D., Zhang, Y. G., Zhang, Z. H.: Bounding sectional curvature along a Kähler-Ricci flow. Comm. Contemp. Math. 11, 1067-1077 (2009) Zbl 1186.53077 MR 2589576

[Ru] Rubinstein, Y. A.: On the construction of Nadel multiplier ideal sheaves and the limiting behavior of the Ricci flow. Trans. Amer. Math. Soc. 361, 5839-5850 (2009) Zbl 1185.53077 MR 2529916

[Se1] Sesum, N.: Convergence of a Kähler-Ricci flow. Math. Res. Lett. 12, 623-632 (2005) Zbl 1087.53063 MR 2189226

[Se2] Sesum, N.: Compactness results for the Kähler-Ricci flow. arXiv:0707.2974

[SeT] Sesum, N., Tian, G.: Bounding scalar curvature and diameter along the Kähler Ricci flow (after Perelman). J. Inst. Math. Jussieu 7, 575-587 (2008) Zbl 1147.53056 MR 2427424

[Shi] Shi, Y. L.: $\alpha$-invariants of smooth cubic surfaces with Eckardt points. Adv. Math. 225, 1285-1307 (2010) Zbl 1204.32014 MR 2673731

[Siu] Siu, Y. T.: The existence of Kähler-Einstein metrics on manifolds with positive anticanonical line bundle and a suitable finite symmetry group. Ann. of Math. 127, 585-627 (1988) Zbl 0651.53035 MR 0942521

[Tian87] Tian, G.: On Kähler-Einstein metrics on complex surfaces with $c_{1}>0$. Invent. Math. 89, 225-246 (1987) Zbl 0599.53046 MR 0894378 
[Tian90] Tian, G.: On Calabi's conjecture for complex surfaces with positive first Chern class. Invent. Math. 101, 101-172 (1990) Zbl 0716.32019 MR 1055713

[Tian90t] Tian, G.: On a set of polarized Kähler metrics on algebraic manifolds. J. Differential Geom. 32, 99-130 (1990) Zbl 0706.53036 MR 1064867

[Tian91] Tian, G.: On one of Calabi's problems. In: Several Complex Variables and Complex Geometry. Proc. Sympos. Pure Math. 52, Amer. Math. Soc., 543-556 (1991) Zbl 0748.53043 MR 1128569

[TV1] Tian, G., Viaclovsky, J.: Moduli spaces of critical Riemannian metrics in dimension four. Adv. Math. 196, 346-372 (2005) Zbl pre02213018 MR 2166311

[TV2] Tian, G., Viaclovsky, J.: Volume growth, curvature decay, and critical metrics. Comment. Math. Helv. 83, 889-911 (2008) Zbl 1154.53024 MR 2442967

[TY] Tian, G., Yau, S. T.: Kähler-Einstein metrics on complex surfaces with $c_{1}>0$. Comm. Math. Phys. 112, 175-203 (1987) Zbl 0631.53052 MR 0904143

[TZ] Tian, G., Zhu, X. H.: Convergence of Kähler Ricci flow. J. Amer. Math. Soc. 20, 575-699 (2007) Zbl 1185.53078 MR 2291916

[TZs] Tian, G., Zhu, X. H.: Perelman's W-functional and stability of Kähler Ricci flow. arXiv:0801.3504

[Wang] Wang, B.: Ricci flow on orbifold. arXiv:1003.0151

[Yau78] Yau, S. T.: On the Ricci curvature of a compact Kähler manifold and the complex Monge-Ampère equation. I. Comm. Pure Appl. Math. 31, 339-411 (1978) Zbl 0369.53059 MR 0480350

[Ye] Ye, R.: Entropy functionals, Sobolev inequalites and $\kappa$-noncollapsing estimates along the Ricci flow. In: ICCM 2007, Vol. II, 218-238

[Zhq] Zhang, Q. S.: A uniform Sobolev inequality under Ricci flow. Int. Math. Res. Notices 2007, no. 17, art. ID rnm056, 17 pp. Z Zbl 1141.53064 MR 2354801 